\title{
A Distributed Resource Block Assignment Scheme for Relay-Assisted Cellular Networks With Self-Organizing TERMinAL RELAYS
}

\author{
by \\ Yaser Mohamed Mostafa Fouad \\ B. Sc. in Electronics and Communications Eng., Arab Academy for Science and \\ Technology and Maritime Transport, \\ Cairo, Egypt, 2009
}

\begin{abstract}
A THESIS SUBMITTED TO THE
Faculty of Graduate Studies and Research

IN PARTIAL FULFILLMENT OF THE REQUIREMENTS FOR THE DEGREE OF

Master of Applied Science in Electrical Engineering
\end{abstract}

Ottawa-Carleton Institute for Electrical and Computer Engineering Department of Systems and Computer Engineering

Carleton University

Ottawa, Ontario

July 2011

(C) Yaser Mohamed Mostafa Fouad, 2011 
Library and Archives
Canada

Published Heritage Branch

395 Wellington Street Ottawa ON K1A ON4 Canada
Bibliothèque et

Archives Canada

Direction du

Patrimoine de l'édition

395 , rue Wellington

Ottawa ON K1A ON4

Canada
Your file Votre référence

ISBN: 978-0-494-83043-7

Our file Notre référence

ISBN: 978-0-494-83043-7
NOTICE:

The author has granted a nonexclusive license allowing Library and Archives Canada to reproduce, publish, archive, preserve, conserve, communicate to the public by telecommunication or on the Internet, loan, distribute and sell theses worldwide, for commercial or noncommercial purposes, in microform, paper, electronic and/or any other formats.

The author retains copyright ownership and moral rights in this thesis. Neither the thesis nor substantial extracts from it may be printed or otherwise reproduced without the author's permission.
AVIS:

L'auteur a accordé une licence non exclusive permettant à la Bibliothèque et Archives Canada de reproduire, publier, archiver, sauvegarder, conserver, transmettre au public par télécommunication ou par l'Internet, prêter, distribuer et vendre des thèses partout dans le monde, à des fins commerciales ou autres, sur support microforme, papier, électronique et/ou autres formats.

L'auteur conserve la propriété du droit d'auteur et des droits moraux qui protège cette thèse. $\mathrm{Ni}$ la thèse ni des extraits substantiels de celle-ci ne doivent être imprimés ou autrement reproduits sans son autorisation.
In compliance with the Canadian Privacy Act some supporting forms may have been removed from this thesis.

While these forms may be included in the document page count, their removal does not represent any loss of content from the thesis.
Conformément à la loi canadienne sur la protection de la vie privée, quelques formulaires secondaires ont été enlevés de cette thèse.

Bien que ces formulaires aient inclus dans la pagination, il n'y aura aucun contenu manquant. 
The undersigned hereby recommend to the Faculty of Graduate Studies and Research acceptance of the thesis

\title{
A Distributed Resource Block Assignment Scheme for Relay-Assisted Cellular Networks With Self-Organizing Terminal Relays
}

\author{
submitted by \\ Yaser Mohamed Mostafa Fouad \\ in partial fulfillment of the requirements for the degree of \\ Master of Applied Science in Electrical Engineering \\ Professor Halim Yanikomeroglu \\ Thesis Supervisor \\ Chair, Department of Systems and Computer Engineering \\ Carleton University \\ July 2011
}




\begin{abstract}
Terminal relaying offers an effective means for improving the performance of OFDMA-based wireless networks. This is because, in addition to offering implementable high orders of spatial diversity to Wireless Terminals (WTs), the number of Terminal Relays (TRs) naturally increases with the number of WTs. However, effective coordination between these TRs becomes a cumbersome task with the increase in the number of TRs. In particular, when their number is large, centralized coordination might not be efficient due to the overhead necessary for Resource Blocks (RBs) coordination. To address this drawback, in this thesis we propose an autonomous resources coordination scheme. In this scheme, the TRs assign RBs to incoming WTs in a way that minimizes the number of hit occurrences at which the same RB is assigned to other WTs.

The set of RBs to be assigned by each TR is endowed with a cyclic group structure. This facilitates sequences generation and enables each TR to have access to the entire set of available RBs. Hence, the proposed scheme is particularly beneficial in terminal relaying systems in which the distribution of the WTs is nonuniform and the Channel Quality Indicators (CQIs) are not available. One problem that arises in the proposed scheme is the complexity involved in selecting the best performing cyclic sequences. To address this problem, we propose an analytical approach in which a metric is employed to systematically select the cyclic sequences. This work is further extended by proposing a graphical cyclic sequences selection technique which significantly reduces the complexity of the selection process. We then exploit the cyclically structured assignment sequences to enhance the performance of the proposed scheme. To do so, we propose an algorithm to utilize the hit occurrences in estimating the load level of the system. This information is then exploited in updating the assignment sequences in order to avoid future hits. Simulation results show that the performance of the proposed scheme is significantly better than that of assignment schemes proposed in the literature.
\end{abstract}




\section{Acknowledgements}

Before beginning, I am extremely grateful to Allah for allowing me to reach this point in pursuing my goal. I am also extremely thankful for my supervisor Prof. Halim Yanikomeroglu for his continuous support and encouragement. I would also like to deeply thank Dr. Ramy Gohary for his generous support along the journey of the Masters. I am also thankful for my colleagues in our research group for their encouragement and insightful suggestions for improving this work.

I would like to express my gratitude for the defence committee Prof. Yuu Ono, Prof. Ivan Stojmenovic and Prof. Changcheng Huang for giving me their precious time to allow me to present my work and for their valuable comments and discussions on improving this thesis.

I would also like to acknowledge Research In Motion (RIM) for financially supporting this research and for the insightful comments to further improve this work.

Finally I dedicate this work to my parents, my brother to whom I owe everything I have reached and for my beloved fiance for her generous support. I cannot be thankful enough for their pure love and guidance in every single moment in my life. 
$\begin{array}{ll}\text { Abstract } & \text { ii }\end{array}$

Acknowledgements $\quad$ iii

Table of Contents $\quad$ iv

List of Figures $\quad$ vi

List of Acronyms viii

List of Symbols $\quad$ ix

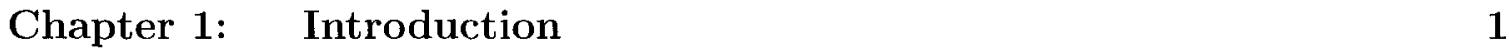

1.1 Relay Assisted Cellular Networks . . . . . . . . . . . . . . . . . 2

1.2 The Resources Assignment Problem . . . . . . . . . . . . . . . . . . . 4

1.3 Thesis Contribution . . . . . . . . . . . . . . . . . . . . . 4

1.4 Published, Submitted and Proposed Manuscripts . . . . . . . . . 7

1.5 Thesis Organization . . . . . . . . . . . . . . . . 8

Chapter 2: $\quad$ Literature Review on Resource Blocks Coordination Schemes 9

2.1 Static Resource Block Assignment Schemes . . . . . . . . . . . . . . . 9

2.2 Dynamic Resource Block Assignment Schemes . . . . . . . . . . . . . 10

2.2.1 Centralized Dynamic Assignment Schemes . . . . . . . . . . . 10

2.2.2 Distributed Dynamic Assignment Schemes . . . . . . . . . . . 11

Chapter 3: Preliminaries 13

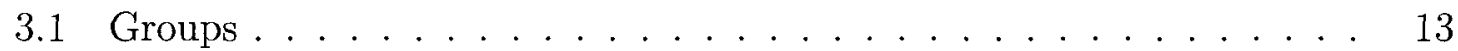

3.2 Cyclic Groups . . . . . . . . . . . . . . . . . . . . . . . . . . . . . . . . . .

3.2.1 Cyclic Additive Groups . . . . . . . . . . . . . . 15

3.2.2 Cyclic Multiplicative Groups . . . . . . . . . . . . . . 16

3.3 Group Isomorphism . . . . . . . . . . . . . . . . . . . . . . . . . . . . . . . . . 17

3.4 The Arithmetic Inverse . . . . . . . . . . . . . . . . . 18

Chapter 4: Problem Definition and System Model 20

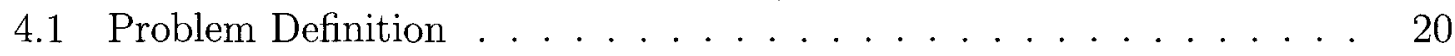

4.2 System Model . . . . . . . . . . . . . . . . . 23 
Chapter 5: $\quad$ A Proposed Solution For A Radio Resource Management Problem $\quad 25$

5.1 The Resource Block Assignment Scheme . . . . . . . . . . . . . 25

5.2 A Metric For Selecting PRCS Pairs . . . . . . . . . . . . . . . . 28

5.2 .1 Computational Complexity of $C(K) \ldots \ldots \ldots$

5.2 .2 Sequence Selection ................. . . 31

5.3 Further Enhancement of the Resource Block Assignment Scheme . . . 31

5.3.1 Hit Source Identification and Avoidance (HIA) . . . . . . . . 32

5.3.2 Computational Complexity of the HIA Algorithm . . . . . . 38

Chapter 6: Insights In Sequences Selection 39

6.1 Group Isomorphism and Proposed Scheme . . . . . . . . . . . . . 39

6.2 Graph Theory and Sequences Selection . . . . . . . . . . . . . 41

6.2.1 A Graphical Selection of PRCS Pairs . . . . . . . . . . . 45

6.2.2 Computational Complexity of The Graphical Solution . . . . . 51

Chapter 7: $\quad$ Simulation Results $\quad 55$

$\begin{array}{lll}\text { Chapter 8: } & \text { Summary and Future Work } & 68\end{array}$

8.1 Thesis Summary . . . . . . . . . . . . . . . . . . . . . 68

8.2 Proposed Future Work . . . . . . . . . . . . . . 70

Appendix A: Proof of Lemma 1 72

$\begin{array}{ll}\text { Appendix B: Proof of Lemma 2 } & 74\end{array}$

$\begin{array}{ll}\text { References } & 77\end{array}$ 


\section{List of Figures}

1.1 A two hop relaying strategy. . . . . . . . . . . . . . . . . 3

1.2 RBs assignment to the BS-Relay and the Relay-WT links. . . . . . . 5

3.1 The Euler Totient function for the first 500 integers. . . . . . . . . . 15

4.1 A two relay example. . . . . . . . . . . . . . . . . 22

5.1 Flow chart of the hit identification and avoidance (HIA) technique. . 34

6.1 One to one mapping between two isomorphic groups. . . . . . . . . 40

6.2 A graphical representation of a cyclic group. . . . . . . . . . . . . 42

6.3 A graphical representation of the cyclic group of order 10 including all its group generators' patterns. . . . . . . . . . . . . . . 43

6.4 a.Cyclic group of order 10 without cyclic shift. b.Cyclic group of order 10 with an anti-clockwise cyclic shift of a step size one $(\mathrm{s}=-1) \ldots . .44$

6.5 A group generator and its inverse of a cyclic multiplicative group of

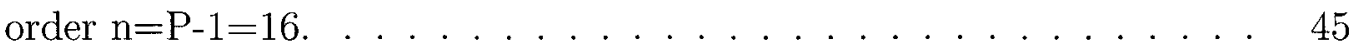

6.6 The graphical notation of the multiplicative cyclic group of order 18 $(P=19)$ with the group generator pair $(2,10)$ being the basis of the circle. . . . . . . . . . . . . . . . . . .

6.7 The graphical representation of the pattern generated from the group generator 3 in the multiplicative cyclic group of order 18. . . . . . 48

6.8 The process of the graphical selection of the PRCS pairs. . . . . . . 52

7.1 Comparison between proposed assignment sequences and the random assignment scheme for $N=P-1$ RBs with $P=17$ and $P=41$. . .

7.2 Comparison between random assignment scheme and the proposed assignment for $N=40$ RBs (i.e., $P=41$ ) with and without HIA and with immediate HIA. . . . . . . . . . . . . . . . . . . . . .

7.3 Comparison between metric minimizing roots and actual average minimizing roots for $P=17$ and $P=127$ with immediate HIA. . . . . 58

7.4 Proposed assignment scheme for $P=17$ and $P=41$ with and without cyclic shifts with immediate HIA. . . . . . . . . . . . . . . . 59

7.5 Comparison between the performance of the PRCS pairs that minimize $\frac{1}{N} \sum_{K=1}^{N} C(K)$ and that of the PRCS pairs that minimize $C(K)$ for each system load $K$. The number of RBs $N=16$. . . . . . . . 
7.6 Comparison between the SINR achieved by the proposed RB assignment sequences and the random assignment sequences for $N=16$ and

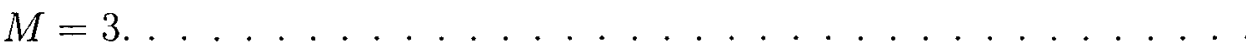

7.7 Comparison between the achievable data rate when the proposed $\mathrm{RB}$ assignment sequences, the random assignment sequences and the optimal centralized assignment scheme with perfect CQI knowledge are employed for $N=16$ and $M=3 \ldots \ldots \ldots \ldots \ldots$

7.8 Comparison between the relative load of the random and proposed assignment sequences at different average threshold SINR levels for $N=16$ RBs.

7.9 Comparison between the PN-based and the proposed assignment schemes for $P=127$ and $n=7$ when the cyclic shifts are set to zero with and without immediate HIA. . . . . . . . . . . . . . . . . . . 


\section{List of Acronyms}

$\begin{array}{ll}\text { AF } & \text { Amplify and Forward } \\ \text { BS } & \text { Base Station } \\ \text { CDMA } & \text { Code Division Multiple Access } \\ \text { CQI } & \text { Channel Quality Indicator } \\ \text { dB } & \text { Decibel } \\ \text { DF } & \text { Decode and Forward } \\ \text { FFR } & \text { Fractional Frequency Reuse } \\ \text { FH } & \text { Frequency Hopping } \\ \text { GADIA } & \text { Greedy Asynchronous Distributed Interference Avoidance } \\ \text { GSM } & \text { Global System for Mobile Communications } \\ \text { HIA } & \text { Hit Identification and Avoidance } \\ \text { OFDMA } & \text { Orthogonal Frequency Division Multiple Access } \\ \text { PN } & \text { PseudoNoise } \\ \text { PRCS } & \text { Primitive-Root-Cyclic-Shift } \\ \text { QoS } & \text { Quality-of- Service } \\ \text { RB } & \text { Resource Block } \\ \text { TR } & \text { Terminal Relay } \\ \text { SINR } & \text { Signal-to-Noise-plus-Interference Ratio } \\ \text { SNR } & \text { Signal to Noise Ratio } \\ \text { TDMA } & \text { Time Division Multiple Access } \\ \text { WT } & \text { Wireless Terminal }\end{array}$




\section{List of Symbols}

\begin{tabular}{|c|c|}
\hline$G$ & A group \\
\hline $\mathcal{Z}_{n}$ & The additive cyclic group of order $n$ \\
\hline$e$ & The identity element in the group \\
\hline$\phi$ & The Euler Totient function \\
\hline$g$ & A group generator of a cyclic group \\
\hline$g^{-1}$ & The arithmetic inverse of the group generator $g$ \\
\hline$P$ & A prime number \\
\hline $\mathcal{F}$ & The isomorphism function \\
\hline$N$ & The total number of resource blocks in the cell \\
\hline$M$ & The number of terminal relays in the cell \\
\hline$K$ & The total number of active wireless terminals in the cell \\
\hline$s$ & The cyclic shift applied to the group generator $g$ \\
\hline$X\left(g_{\imath}, s_{\imath}\right)$ & The load matrix of relay $i$ that is assigned the PRCS pair $\left(g_{l}, s_{2}\right)$ \\
\hline & The pairwise hit matrix between relays $i$ and $j$ \\
\hline,$\left.k_{M}\right)$ & $\begin{array}{l}\text { The total number of hits observed by } M \text { RTs loaded by loads } \\
\left(k_{1}, \ldots, k_{M}\right)\end{array}$ \\
\hline$C(K)$ & The metric for selecting the PRCS pairs \\
\hline $\mathcal{S}_{K}$ & The number of ways an integer can be partitioned into $M$ ordered parts \\
\hline$\left(g, g^{-1}\right)$ & A pair consisting of a group generator and its inverse \\
\hline $\mathcal{U}$ & The set of group generator pairs \\
\hline IEI & Intersection element of interest \\
\hline$V_{I E I, c_{1}, c_{2}}$ & $\begin{array}{l}\text { The total number of hits observed over all possible load combinations } \\
\text { given the occurrence of an intersection at the IEI located at } c_{1} \in s e q_{1} \\
\text { and at } c_{2} \in \text { seq }_{2}\end{array}$ \\
\hline $\operatorname{seq}$ & The sequence generated by the group generator $g$ and the cyclic shift $s$ \\
\hline & $\begin{array}{l}\text { The total number of hits observed over all possible load combinations } \\
\text { between the sequences } s e q_{1} \text { and } s e q_{2}\end{array}$ \\
\hline$I_{\text {total }}$ & $\begin{array}{l}\text { The total number of hits observed over all possible load combinations } \\
\text { between all the pairs of selected group generators }\end{array}$ \\
\hline
\end{tabular}




\section{Chapter 1}

\section{Introduction}

Wireless communications technologies are undergoing an evolution as compact communication devices continue to have more processing capabilities and lower production costs. The presence of such devices allowed a larger spectrum of the society to have access to these technologies and hence introduced a wide variety of applications. Some examples would be multimedia streaming, security, remote sensing and electronic commerce.

The introduction of these applications generated a continuously increasing demand for high data rate communications to fulfil their information exchange requirements [1]. This demand, stimulated pressure to develop the currently available communication systems so that they become able to meet the desired data rates for information exchange.

Unlike wired systems, a communication system operating over a wireless channel posses the attractive feature of mobile connectivity between devices without requiring the deployment of costly wired infrastructure. In addition, there are scenarios where wireless systems can even become the sole solution for communications when the terrain makes the deployment of wired infrastructure infeasible. Hence motivated by the offered flexibility and mobility, the trend for data communications shifted towards wireless networks as convenient means for data communications. 
When designing a wireless communication system to meet this demand, the limited capabilities of the wireless link should be taken into consideration [2]. This is because when the communication signal propagates through the environment it becomes susceptible to several channel impairments that affect the quality of the signal. For example, the signal might be blocked due to the presence of an obstacle between the transmitter and the receiver and hence resulting in a poor signal quality at the receiver side. In addition to channel impairments, the signal power suffers from attenuation as it propagates through the wireless channel due to signal dispersion. This attenuation is inversely proportional to the frequency at which these signals are transmitted. Hence, although the available spectrum (the band over which data can be communicated) in the environment is infinite, only a limited portion is suitable for wireless data communications with large coverage area requirements.

In order to satisfy the wireless high data rate communications requirements, the system designer should focus on improving the link quality between the transmitter and the receiver so that more data can be accommodated without exceeding the tolerable error level. One possible solution for link quality improvement would be to employ a two-hop communication strategy [3]. In such a scenario, the low quality communication link between the transmitter and the receiver is replaced by two links of higher quality as illustrated in Figure 1.1.

\subsection{Relay Assisted Cellular Networks}

A relay is designed to be smaller than the Base Station (BS) in terms of size and to have limited processing capabilities. These relaying devices are wirelessly connected to the backhull of the network and hence in addition to their low cost, they can be easily deployed in strategic locations. In particular, fixed relays are positioned 


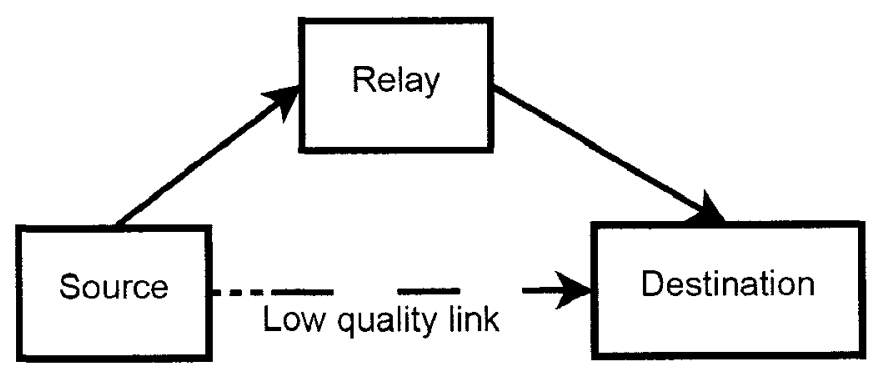

Figure 1.1: A two hop relaying strategy.

in a relatively good location, from the perspective of signal propagation, between transmitters and receivers. Moreover the employment of relays in cellular network enriches the concept of spatial diversity as an additional degree of freedom. Hence the deployment of relays is expected to improve the link quality between the BS and the Wireless Terminals (WTs) rendering a communication network with either extended coverage [4] or higher data rate transmissions [5]. This can be done by employing a higher modulation level (embedding more bits in each transmitted symbol) or by reducing the coding level and hence more user data can be transmitted.

In addition to fixed relays which have static locations, limited numbers and are operated by the service provider, there is another category of relays known as Terminal Relays (TRs). In such a scenario, an idle WT that is not engaged in a voice or a data call can be utilized to relay active WTs data streams in a cooperative fashion. Due to their random distribution, it is more likely to have a TR that has good signal quality within the premises of the WTs and hence can significantly improve the network performance. However, unlike fixed relays, TRs are not operated by the service provider, continuously update their status as they alternate between active and relaying modes and also frequently update their locations as users continue to move from one location to another. 


\subsection{The Resources Assignment Problem}

In a wireless communication system, a Resource Block $(\mathrm{RB})$ can be defined as the smallest portion of the available physical resource that can be assigned to a single link for data transmission. This RB can either be a specific part of the spectrum (a

number of subcarriers) in Orthogonal Frequency Division Multiple Access (OFDMA), a specific time interval in Time Division Multiple Access (TDMA) or even a given code in Code Division Multiple Access (CDMA). When an RB is assigned to a WT, it starts to transmit its data streams in this assigned dwell only. By definition, each $\mathrm{RB}$ is orthogonal on the other RBs and hence the data streams transmitted on any $\mathrm{RB}$ will be invisible, from an interference perspective, to one another.

Due to interference limitations, a relay has to work in a half-duplex mode and cannot transmit and receive on the same RB simultaneously. Hence, the two hop link between the BS and the WT will need to be assigned one RB for each of the BS-Relay and the Relay-WT links as illustrated in Figure 1.2.

This increase in the number of required RBs for transmission in addition to their limited availability (the spectrum is considered to be a very scarce commodity), raise a concern on how the RBs can be efficiently assigned in order to meet the desired data rate requirements.

\subsection{Thesis Contribution}

In this thesis, we consider the problem of $\mathrm{RBs}$ assignment in relay-assisted OFDMA-based cellular systems that employ a terminal relaying strategy. Several schemes have been devised in literature to coordinate the assignment of resources between the network elements. To the best of our knowledge, the available schemes 


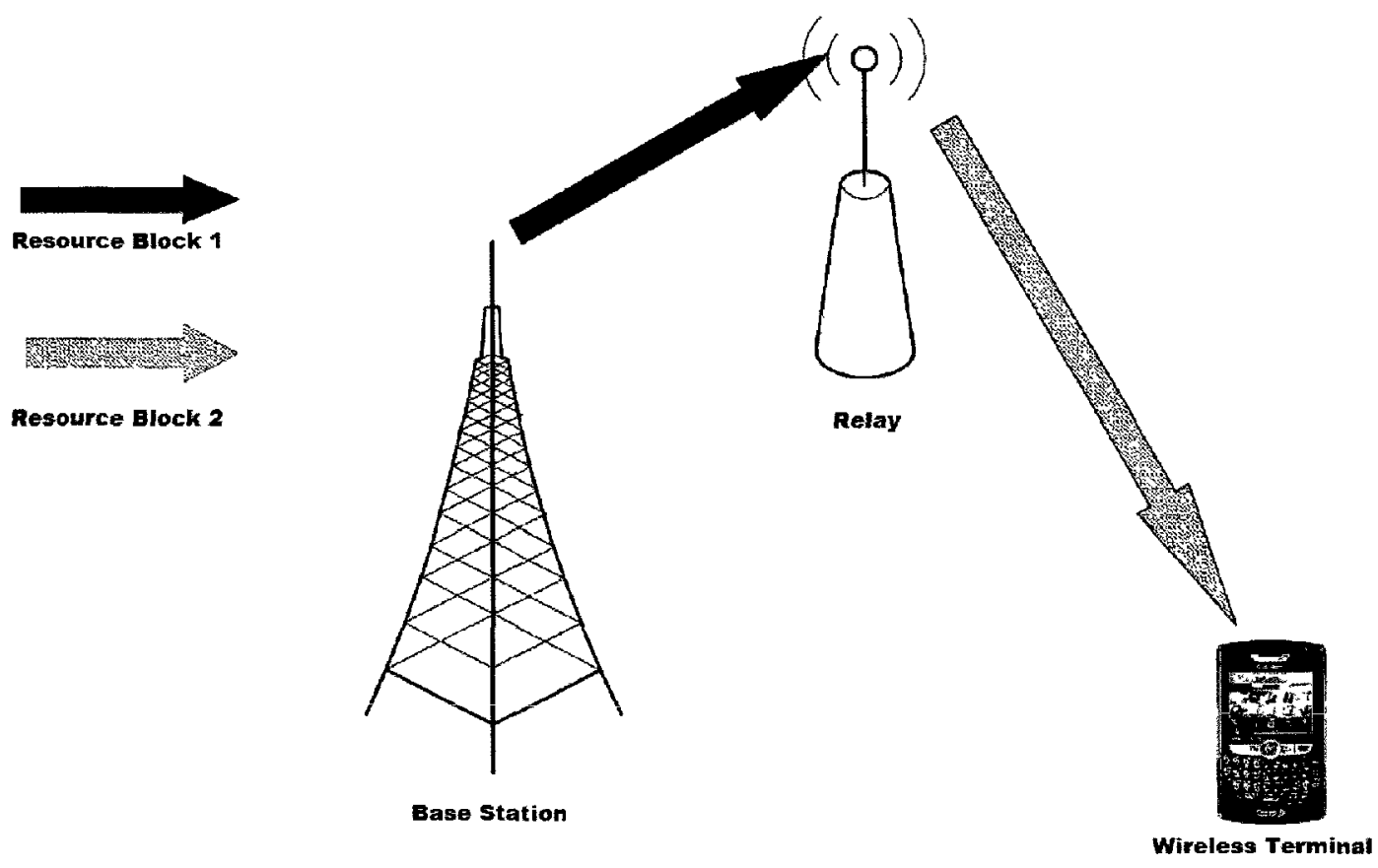

Figure 1.2: RBs assignment to the BS-Relay and the Relay-WT links.

in literature depend on the presence of the CQIs either at a central coordinating entity when a centralized RBs assignment strategy is employed or at each TR in the distributed assignment schemes. However, in relay-assisted cellular systems, it may be impractical to adopt a centralized resources coordination scheme due to its associated coordination overhead. Moreover, rapid channel variations may cause the WTs to frequently update their serving TRs, resulting in rapid variations in the network topology. These variations render the timely acquisition of the CQI information difficult and impede the implementation of centralized CQI-dependent assignment schemes.

A more practical approach is to assign the RBs in a distributed manner whereby TRs autonomously select the RBs of the WTs they assist. However, in the absence 
of centralized coordination this strategy may result in undesirable instances at which one RB is used by many WTs while other RBs are not used. Such instances result in high, yet avoidable, intra-cell interference. In particular, we propose a scheme for minimizing intra-cell interference by reducing the number of hit occurrences; that is, occurrences at which an RB is assigned to multiple WTs.

Unlike other schemes available in literature, the scheme proposed herein is autonomous in the sense that the assignment of RBs by a TR is distributed and independent of the CQIs. Hence, the proposed scheme is particularly suited for systems employing a terminal relaying strategy. Moreover, the autonomous nature of the proposed scheme also enables more efficient exploitation of the available spectrum than centralized schemes. It is assumed that each TR is entitled to use as many RBs as needed; a feature that enables the TRs to efficiently adapt to bursty traffic and nonuniform WTs distributions without coordination. To achieve this goal, the set of available RBs is endowed with a cyclic group structure (illustrated in Chapter 3) that allows each TR to generate its own sequence to be followed when assigning its RBs. The adoption of the cyclic structure not only facilitates the generation of the assignment sequence at each TR with minimum communication overhead but also enables each TR to avoid future hits. For this to be achieved, we propose a novel algorithm in Chapter 5 to utilize the knowledge obtained when a hit occurs in order to avoid future hits. In this algorithm, the structure of the assignment sequences is exploited to acquire information about the load level of other TRs in the network when a hit occurs. This knowledge is then utilized in updating the assignment sequences adopted by the TRs to improve the performance.

Due to the availability of a large number of cyclically generated sequences it becomes difficult to perform an exhaustive search over all possible combinations of 
sequences to find the ones providing the best performance. Hence, in this thesis we provide a metric for faster selection of the best performing cyclic sequences in Chapter 5. This work is further extended by providing a graphical selection technique that renders a faster choice of cyclic sequences in Chapter 6. We also note that despite the fact that the scope of our proposed work was on relay assisted cellular networks, the proposed assignment scheme is still extensible to other wireless networks. This includes any wireless network that requires a distributed assignment of resources among its elements in the absence of centralized coordination and CQIs. Some examples would be the Wireless Fidelity (WiFi) and wireless sensor networks.

\subsection{Published, Submitted and Proposed Manuscripts}

- Yaser Fouad, Ramy Gohary, and Halim Yanikomeroglu, "A Resource Block Assignment Scheme For OFDMA-Based Cellular Networks With Self-Organizing Terminal Relays," in Proc. IEEE Vehıc. Tech. Conf. (VTC2011-Spring), (Budapest), May 2011.

- Yaser Fouad, Ramy Gohary, and Halim Yanikomeroglu, "An Autonomous Resource Block Assignment Scheme For OFDMA-Based Relay-Assisted Cellular Networks", under review in IEEE Trans. Wrreless Commun. (submission: 18 October 2010, 1st results: 01 January 2011, 1st review submitted: 01 March 2011).

- Yaser Fouad, Ramy Gohary, and Halim Yanikomeroglu, "Graph Theory And Its Applications In Resource Block Assignment Schemes For Relay-Assisted Cellular Networks", to be submitted to the IEEE Trans. Wireless Commun. 


\subsection{Thesis Organization}

The remainder of this thesis is organized as follows. In Chapter 2 we provide a literature survey on proposed resource block coordination schemes for wireless networks. In this chapter, some of the proposed schemes which fall in the context of our work are categorized and presented. Unlike Chapter 2, in Chapter 3 we introduce some of the basic concepts of number theory. In particular, we introduce some of the key concepts of group theory that will be exploited in the design of our proposed scheme. This introduction is then followed by the problem definition and system model adopted in this thesis in Chapter 4. We then introduce our proposed RB assignment scheme which is the scope of our work in Chapter 5. This work is further extended in Chapter 6 where we introduce a graphical cyclic sequences selection technique. The performance of the proposed scheme is then evaluated through simulations in Chapter 7 . We conclude this thesis in Chapter 8 by highlighting the key points in our work and introducing the open research directions for future work. 


\section{Chapter 2}

\section{Literature Review on Resource Blocks Coordination Schemes}

The concept of relaying in cellular networks is considered to be one of the promising candidates to achieve ubiquitous high-throughput coverage throughout the cell. However the added complexity to the network raises a concern on how this additional degree of freedom can be efficiently utilized. In particular, a major concern is how the RBs assignment process can be coordinated among the network elements so that mutual interference can be minimized without affecting the data throughput.

The problem of resources coordination has been extensively studied in literature. Several schemes have been devised for resource block coordination and even though some of these schemes are not dedicated to the relay-assisted networks, they are still extensible to fit the relaying environment. In our point of view, the available literature on resources assignment can be divided into two main categories. Both categories will be discussed in the following sections.

\subsection{Static Resource Block Assignment Schemes}

In this category of schemes, the assignment of resources is static and hence independent of the load levels of the cells and the CQIs. One example would by the cluster structure for frequency reuse adopted in the Global System for Mobile communications (GSM) [6]. In this scheme, each RB cannot be reused within a specific 
distance in order to reduce inter-cell interference. Another example would be what is known as Fractional Frequency Reuse [7]. In this work, the available resources are divided into two partitions. The first partition is used with a frequency reuse level of 1 and limited to the center of the cell. On the contrary, the second partition is used with a lower frequency reuse level and is utilized by the users located at the periphery of the cell.

\subsection{Dynamic Resource Block Assignment Schemes}

Unlike the first category, these RBs coordination schemes adopt a dynamic RBs assignment strategy. In particular, the assignment of RBs changes with time in order to optimize the network performance. Such schemes can be further divided into centralized and distributed based upon the presence of a central coordination entity (usually located at the BS). However, a major difference between these schemes and our work is that they assume perfect knowledge of the CQIs. This information is assumed to be available either at the central coordinating entity or at the WTs and TRs in the case where we have distributed resources assignment.

\subsubsection{Centralized Dynamic Assignment Schemes}

In these schemes, the assignment of RBs to the network elements is performed centrally by the BSs. In [8], the authors aimed to improve the throughput by exploiting spatial diversity. In this work, they maintained a specific diversity level by assigning a minimum relaying requirement for each TR. As another approach, the authors in [9] considered the power allocation problems in relay-assisted cellular networks. They assumed that the WTs which are close to the BS transmit at significantly lower power levels when compared to the users at the periphery of the cell 
and hence can collaborate to relay the signals of cell-edge WTs. They provided an efficient joint optimization solution for selecting both the effective relaying terminals and the relaying strategies to be employed in addition to the allocation of resources for network elements. Another centralized RBs coordination scheme was introduced in [10]. In this paper, the authors introduced a hierarchical OFDMA transmission protocol in which all devices employing one hop communications represent the core network while the two hop communicating devices constitute a sub-network. An efficient convex optimization algorithm is then employed to optimize the assignment of the RBs for optimal resources allocation in terms of aggregate cell throughput. However, due to their centralized structure, it might not be feasible to exploit such schemes in a terminal relaying environment with a significantly large number of TRs due to its associated overhead.

\subsubsection{Distributed Dynamic Assignment Schemes}

Distributed RBs assignment schemes are considered as promising solutions to achieve efficient resources coordination in the terminal relaying environment. One example would be the scheme proposed in [11]. When this scheme is applied in relay-assisted networks, each TR chooses its initial assignments randomly and independently of other TRs. However when the CQIs become available the assignment of RBs is then updated to improve the cell performance. Another example would be the Frequency Hopping (FH) schemes in which the WTs hop across frequency slots. In these systems, the frequency slot occupied by each WT at a given dwell interval is determined by a prescribed PseudoNoise (PN) based hopping pattern. In such schemes, the hopping patterns are continuously updated based on the CQIs in order to improve the network throughput; see e.g., [12-15]. 
On the contrary, in [16], a game theoretic approach is adopted. In this scheme, WTs and TRs represent the players which compete to acquire the available RBs. Similarly, the authors in [17] considered the same approach, however they assumed that the RBs assignment lasts for relatively long time. Hence they modeled the RBs assignment process as multiple games or rounds in which the players remember their previous choices. They also applied a power based punishment on WTs which are causing a significantly large interference level.

In [18], B. Babadi and V. Tarokh proposed a Greedy Asynchronous Distributed Interference Avoidance (GADIA) algorithm. In their work, they assumed a canonical network model where agents are divided into clusters and each cluster is represented by its cluster head. The proposed scheme achieved comparable performance to the optimal centralized resources assignment. However as illustrated earlier, the scheme assumes the presence of the CQIs at the relays. Another proposed approach appears in [19]. In this paper, the authors tackled the problem of TRs selection and RBs assignment using an auction-based strategy. In such a scenario, the WTs compete by bidding for choosing their RBs. The proposed scheme employs two types of auctions. The first is a power based auction in order to efficiently allocate the resources and the second one is an SNR based auction for enforcing fairness among WTs. Finally a priority based approach was adopted in [20]. In this paper, the RBs assignment is based on the Signal-to-Interference-plus-Noise-Ratio (SINR). However, an assignment priority is given to the WTs that can achieve diversity gain when terminal relaying is employed. 


\section{Chapter 3}

\section{Preliminaries}

In this thesis, we propose an autonomous $\mathrm{RB}$ assignment scheme for relay-assisted cellular networks. As illustrated in the introduction, the proposed assignment sequences are endowed with a cyclic group structure to facilitate the assignment of RBs. Hence in this chapter, we introduce some of the basic elements of group theory $[21-23]$.

\subsection{Groups}

A group is a set $G$ on which a group operation (denoted by juxtaposition) is defined such that the following closure property is satisfied: For all $(x, y) \in G \times G$ the element $x y \in G[21]$. Additionally, the following properties should also be satisfied:

- The presence of an identity element in the group; There is an element $e \in G$ such that $e x=x e=x$ for each element $x \in G$.

- The presence of an inverse for every element in $G$; For every element $x \in G$ there exists an element $y$ such that $x y=y x=e$

- Associativity such that for each $x, y, z \in G,(\mathrm{xy}) \mathrm{z}=\mathrm{x}(\mathrm{yz})$. 
As an example, we can consider the set $S=\{0, \ldots, n-1\}$ which forms a group under modulo- $n$ addition. This is because in addition to the associative group operation, there exists an identity element $e=0$ and an inverse for each element $x$ which is $(n-x)$ where $x+(n-x)(\bmod n)=e$.

There exists a wide variety of subgroups such as symmetry groups, permutation groups and cyclic groups. However, in this chapter we will focus only on cyclic groups which represent the foundation of our proposed scheme.

\subsection{Cyclic Groups}

One subdivision of groups is cyclic groups which are characterized by being the only groups that can be generated from a single element such that when a specific operation (noted as the group operation) is performed on this element, all the elements of the group are generated in a unique sequence. Such an element is known as a group generator.

For a group of $n$ elements (the order of the group is $n$ ), the number of group generators is given by the Euler Totient function, $\phi(n)$, which is defined as follows:

Definition 1. The cardinality of the set of integers that are coprime with $n$; that ıs, $\phi(n)$ us the cardınaluty of the set $\{q<n \mid \operatorname{gcd}(q, n)=1\}$, where $\operatorname{gcd}(q, n)$ is the greatest common divisor of $q$ and $n$ [21].

Although the Euler Totient function, $\phi(P-1)$, is not monotonically increasing, it is known that its value is large for large primes, e.g., $\phi(100)=40, \phi(310)=120$, and $\phi(498)=164$. For illustration, a plot of $\phi(P-1)$ is given in Figure 3.1.

There are different types of cyclic groups depending on the group operation. As an example we will consider the additive and multiplicative cyclic groups. 


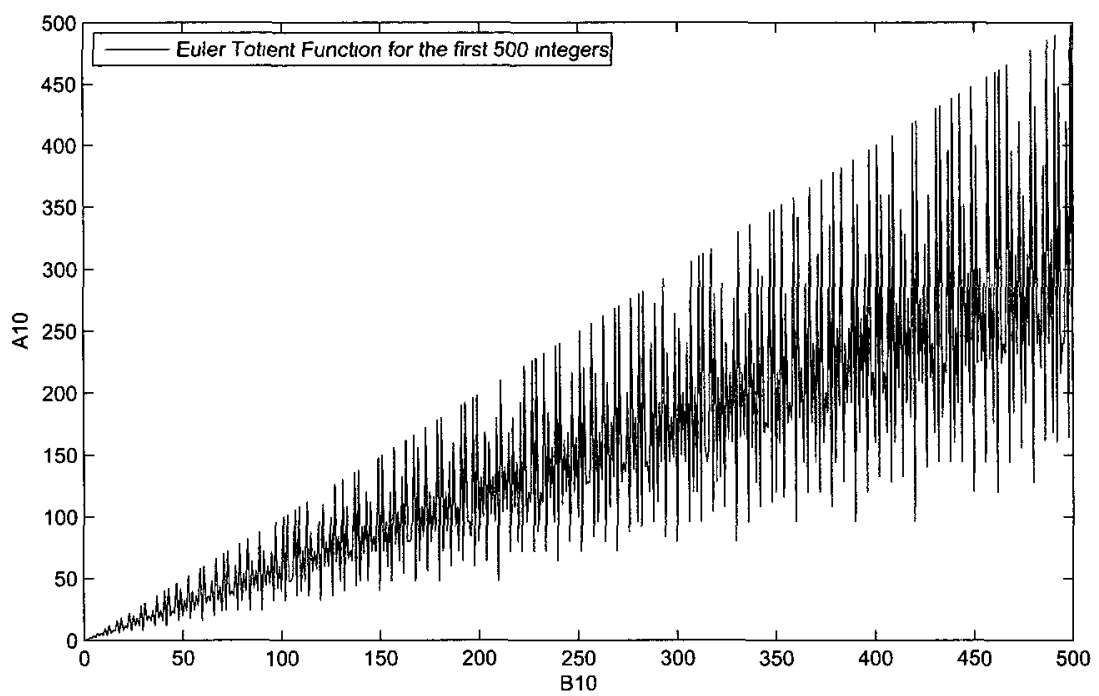

Figure 3.1: The Euler Totient function for the first 500 integers.

\subsubsection{Cyclic Additive Groups}

A cyclic additive group is a cyclic group with modulo- $n$ addition as the group operation. In this case, based on the group definition stated above, for every $x, y \in G$, $x+y(\bmod n) \in G[21]$. For example, the set $\{0, \ldots, n-1\}$ forms a cyclic additive group under modulo- $n$ addition. In this example, the number of group generators is given by $\phi(n)$, as illustrated hereinabove, and for each group generator $g$ the ordered set

$$
\left((g)\left(k_{1}\right) \quad(\bmod n), \ldots,(g)\left(k_{n}\right) \quad(\bmod n)\right)=(0, \ldots, n-1)
$$

for some integers $k_{\imath} \in\{1, \ldots n\}, i=1, \ldots, n$. For illustration, when $n=6$ there are $\phi(6)=2$ group generators which are $g_{1}=1$ and $g_{2}=5$. According to 3.1, the sequences generated by these group generators would be $(1,2,3,4,5,0)$ and $(5,4,3,2,1,0)$, respectively. 


\subsubsection{Cyclic Multiplicative Groups}

A cyclic multiplicative group is a cyclic group in which the group operation is modulo- $n$ multiplication. That is, for every $x, y \in G, x y(\bmod n) \in G$. A classic result in number theory asserts that the set $\{1, \ldots, n-1\}$ forms a cyclic multiplicative group under modulo- $n$ multiplication if and only if $n$ is a prime number [21].

Let $P$ be a prime number. A primitive root of $P$ is defined to be an integer $g \in\{1, \ldots, P-1\}$ such that the ordered set

$$
\left(g^{k_{1}} \quad(\bmod P), \ldots, g^{k_{p-1}} \quad(\bmod P)\right)=(1, \ldots, P-1)
$$

for some integers $k_{\imath} \in\{1, \ldots P-1\}, i=1, \ldots, P-1$. The number of primitive roots is given by $\phi(P-1)$, and as illustrated hereinabove each primitive root by definition generates all integers that are coprime to $P$ which in this case would be $(1, \ldots, P-1)$. Hence, it can be concluded that the group generators of the group $G=\{1, \ldots, P-1\}$ are the primitive roots of the prime number $\mathrm{P}$.

There is no specific formula for computing the primitive roots of a prime number. However they can be found by efficient techniques. For example, in the case of relatively small primes, primitive roots can be obtained by searching for $g \in\{1, \ldots, P-1\}$ such that the sequence $\left\{g^{k}(\bmod P)\right\}_{k=1}^{P-1}$ spans all integers from 1 to $P-1$. The primitive roots are also tabulated for the first few thousand prime numbers. As an illustrative example, when $P=7$ there are $\phi(6)=2$ primitive roots which are $g_{1}=3$ and $g_{2}=5$. Direct evaluation of $g_{\imath}^{k_{1}}(\bmod P), k_{1}=1, \ldots, 6, i=1,2$ yields the sequences $(3,2,6,4,5,1)$ and $(5,4,6,2,3,1)$ respectively.

Finally, we present our novel result that will be used to expand the set of sequences that can be cyclically generated by primitive roots: 
Lemma 1. Let $G$ be a cyclic multiplicative group and let $g_{1}$ and $g_{2}$ be two distinct generators modulo-P. Then, there is no cyclic shift for which the sequence generated by $g_{1}$ coincides with the sequence generated by $g_{2}$.

Proof. See Appendix A.

This result implies that cyclic shifts enrich the design of sequences by an additional degree of freedom that cannot be obtained from primitive roots alone.

\subsection{Group Isomorphism}

An isomorphism is a function $\mathcal{F}$ from a group $G$ to a group $\bar{G}$ that satisfies the following properties:

- One to one mapping. That is for every element $x_{1}, x_{2} \in G$ if $\mathcal{F}\left(x_{1}\right)=\mathcal{F}\left(x_{2}\right)$ then $x_{1}=x_{2}$.

- Onto. That is for every element $y \in \bar{G}$ there exists an element $x \in G$ such that $\mathcal{F}(x)=y$

- Preservation of the group operation. For example, if the mapping is done from a group $G$ with a multiplicative group operation to a group $\bar{G}$ with an additive group operation then $\mathcal{F}(x y)=\mathcal{F}(x)+\mathcal{F}(y)$ where $x$ and $y$ are elements in $G$ and $\bar{G}$ respectively.

If two groups, $G$ and $\bar{G}$, are isomorphic then they both have the same algebraic structure and properties.

A basic result in group theory asserts that all cyclic groups of order $n$ are isomorphic to the additive cyclic group of order $n, \mathcal{Z}_{n}$, irrespective of the group opera- 
tion [22]. Additionally, we present the following lemma which will be used to confine the scope of our work.

Lemma 2. Given that $G$ and $\bar{G}$ are two cycluc groups of order $n$, then the sequence generated by $g_{1} \in G$ us equvvalent to a sequence generated by a $g_{2} \in \bar{G}$ under the ısomorphısm $\mathcal{F}\left(g^{k}\right)=k$ where $g$ us one of the group generators of $G$.

Proof. See Appendix B.

\subsection{The Arithmetic Inverse}

In modular arithmetic the modular multiplicative inverse of an integer $x$ modulo $n$ is represented by $x^{-1}$ and can be defined as follows:

$$
x x^{-1} \equiv 1 \quad(\bmod n)
$$

This inverse $x^{-1}$ exists if and only if $x$ and $n$ are coprime. For illustration, if we consider the values of $x$ and $n$ to be 3 and 17 respectively then the arithmetic inverse $x^{-1}=6$ this is because

$$
3 \times 6 \equiv 1 \quad(\bmod 17)
$$

Accordingly, when a multiplicative cyclic group of order $P-1$ is considered, there exists a unique inverse for each group generator $g$ and its value is given by the following equation:

$$
g^{-1}=g^{P-2} \quad(\bmod P)
$$

This is because, for any group generator $g$ of the multiplicative cyclic group of order $P-1$ we can prove that the following equation holds:

$$
g^{P-1} \equiv 1 \quad(\bmod P) .
$$


To prove the previous equation, let $g^{z}$ be the modular arithmetic inverse of the group generator $g$ then, from Equations 3.3 and 3.5 we have:

$$
g g^{z} \equiv g^{P-1} \equiv 1 \quad(\bmod P)
$$

This concludes that,

$$
\begin{gathered}
z+1=P-1 . \\
z=P-2 .
\end{gathered}
$$




\section{Chapter 4}

\section{Problem Definition and System Model}

In this chapter, we start by presenting the motivation behind our work. In particular, we illustrate the considered radio resource management problem for relay assisted cellular networks. This is then followed by another section in which we identify the system model adopted throughout the thesis.

\subsection{Problem Definition}

Fixed relays, as illustrated in Chapter 1, are expected to enhance the performance of cellular networks. However, their efficacy becomes less pronounced with the increase in the number of WTs with high data rate requirements. This is because with the increase in the number of WTs, maintaining a certain quality of service requires increasing the number of fixed relays which subsequently increases the cost of the network and the coordination overhead. These difficulties can be mitigated by employing the terminal relaying scheme discussed in Chapter 1.

In a terminal relaying environment, idle terminals relay the signals of active ones to improve the performance of the network. Since, at a given time instant, a considerable portion of WTs is likely to be idle, the prospective increase in the overall number of WTs will result in a large number of TRs. However, the large number of these terminals and the incidental manner in which they are likely to operate renders 
their coordination significantly more cumbersome than the coordination of their fixed counterparts. Hence, it can be seen that although terminal relaying overcomes some of the limitations of fixed relaying, it requires significantly more efficient coordination mechanisms. Therefore, a problem that arises in relay-assisted communication systems is how the RBs are assigned to the set of WTs that are assisted by a particular relay.

When the relays are fixed and the CQIs are available, the BSs can assign the RBs in such a way that optimizes the network performance. However, this task becomes inefficient in terminal relaying scenarios with large numbers of TRs. In these situations, a more practical approach is to assign the RBs in a distributed manner whereby TRs autonomously select the RBs of the WTs they assist. In such a scenario, the TRs will not be able to identify the RBs that can be reused without incurring high intra-cell interference. This is because when a particular RB is assigned to multiple WTs, the intra-cell interference levels observed by these WTs increase, resulting in a significant deterioration of their signal quality. Such an occurrence is undesirable and will be referred to as a hit. Assuming that the number of WTs does not exceed the number of available RBs, the objective of each TR then becomes to assign the RBs in a way that minimizes the number of hit occurrences.

For illustration, in Figure 4.1 we provide a two-TRs example for an RB assignment scheme that yields a minimum number of hits. Assume that each TR has access to the same set of $N$ RBs. We will denote this set by $\mathcal{R}=\left\{r_{1}, \ldots, r_{N}\right\}$. The first TR assigns the RBs in an ascending order whereby its $k_{1}$-th incoming WT is assigned the $\mathrm{RB} r_{k_{1}} \in \mathcal{R}$. In contrast, the second TR assigns the RBs in a descending order whereby its $k_{2}$-th incoming WT is assigned the RB $r_{N-k_{2}+1} \in \mathcal{R}$.

From this simple assignment scheme it is seen that a hit will occur if and only 

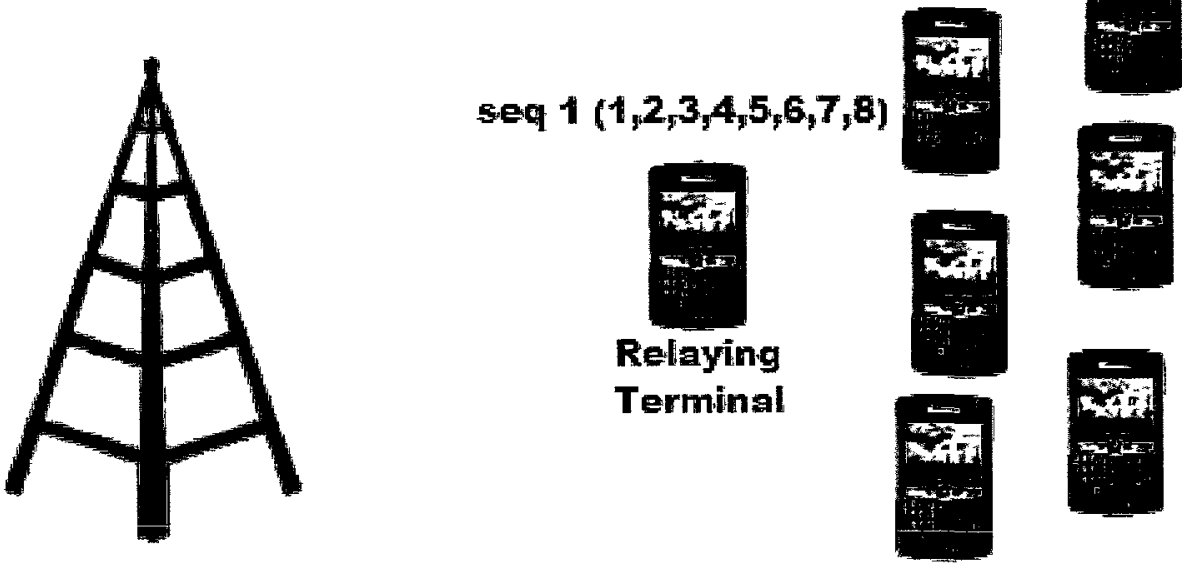

seq $2(8,7,6,5,4,3,2,1)$

Active Terminals

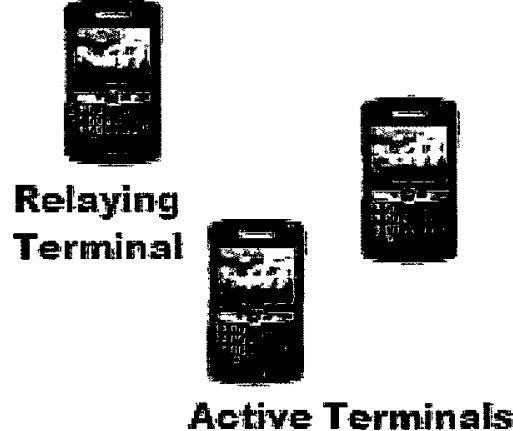

Figure 4.1: A two relay example.

if the total network load $K=k_{1}+k_{2}>N$. This is because when $K=N$, all the available RBs will be assigned to the WTs. Hence, assigning an RB to any additional incoming WT will result in the occurrence of a hit.

Unfortunately, in contrast with the case of $M=2$, when the number of TRs $M>2$, it is easy to show that there is no scheme that can eliminate the possibility of hits without compromising the availability of the $N$ RBs to all TRs. In order to address this difficulty, in the next chapter we will introduce our proposed scheme 
that enables the TRs to assign the RBs in a way that results in a relatively small number of hits. To enhance our scheme, we assume that the TRs are able to detect hit occurrences and utilize this information in avoiding future hits according to the algorithm proposed in Chapter 5.

\subsection{System Model}

We now describe the system model adopted in this work. We consider a relayassisted OFDMA-based cellular network in which a terminal relaying strategy is employed. In our proposed system, it is assumed that WTs switch to the relaying mode as soon as they become idle and hence, no centralized designation by the BS is required. Each BS has $N$ RBs at its disposal, is assisted by $M$ TRs, and serves $K$ active WTs. Each TR has access to all the $N$ RBs of the BS.

The assignment of RBs for the BS-TR links is assumed to be performed by the BS in a centralized manner, which is a reasonable assumption given the typically high processing capabilities of the BS. Hence, our focus herein is on the autonomous assignment of RBs for communication over the TR-WT and WT-TR links. By restricting our attention to the case in which $N=P-1$, where $P$ is a prime integer, we will be able to invoke the cyclic group structure described in Chapter 3. Note that, in general, this assumption is not overly restrictive because primes are denser than squares in the set of integers [23]. For the case in which the number of RBs in the system $N$ is not equal to a prime minus one, the closest prime $P \geq N+1$ can be used and the entries larger than $N$ can be skipped in the assignment. In this case, it is also possible to employ other group structures; e.g., the group structure used for generating PN sequences. However, those group structures are typically more complicated to generate and optimize. For instance, PN-based assignments require 
the search over primitive polynomials. In contrast, for cyclic multiplicative groups, it is only required to take modulo- $P$ of the powers of the primitive roots of $P$.

The WTs access the system one at a time according to a counting process [24]; i.e., the number of WTs in the entire system increases at succedent discrete time instants. This assumption is necessary to ensure that the first TR to assign a particular RB retains it when an assignment conflict occurs. In this situation, the conflicting TRs will refrain from using this RB. However, each TR can still be loaded by an arbitrary number of WTs independently of the load of the other TRs. Furthermore, it will be observed in the RB assignment scheme described hereinafter that the WTs to be served by the same TR can access the system simultaneously. Using this observation, it is possible to assign multiple RBs to WTs with high traffic requirements.

A TR is selected by the incoming WT depending on its observed signal strength. However, as the wireless channel between the TR and the WT undergoes rapid variations, this choice is frequently updated resulting in a highly dynamic network topology. Furthermore, the backhaul link between the TRs and the BS also contribute to the variations in the network topology. For example, if a TR experiences deep fading on the backhaul link, both the BS and the TR will be able to detect this occurrence and the TR will subsequently notify the WTs to update their TR selection. The TRs assign the RBs to the incoming WTs without knowing their respective channel quality indicators (CQIs). 


\section{Chapter 5}

\section{A Proposed Solution For A Radio Resource Management Problem}

We begin this chapter by introducing the proposed RB assignment scheme in the first section. This is then followed by the proposed metric that facilitates a more efficient utilization of the proposed scheme. This chapter is then concluded by the last section in which we introduce our proposed hit identification and avoidance algorithm to further improve the performance of the proposed scheme.

\subsection{The Resource Block Assignment Scheme}

Assume that the communication between the BS and the WTs is assisted by $M \leq \phi(P-1)$ TRs, where $P$ is a prime integer. Furthermore, we make the mild assumptions that the number of available RBs is $P-1$ and that the number of WTs, $K \leq P-1$; cf. Chapter 4 . In the proposed scheme, each TR $i \in\{1, \ldots, M\}$ uses a group generator $g_{i}$, which is one of the $\phi(P-1)$ primitive roots of $P$; cf. Chapter 3 . If the TRs operate in a decode-and-forward (DF) mode, the group generator can be assigned by the BS and embedded in the first BS-TR communication. Alternatively, if the TRs operate in an amplify-and-forward mode, they will not be able to decode the BS signals. In this case, the group generators can be autonomously selected by the TRs from locally built-in look-up tables. The latter strategy can be also used for TRs employing DF relaying. Whether the group generators are provided by the BS or 
locally selected from look-up tables, providing the TRs with these generators incurs negligible resource consumption and requires no further monitoring from the BS. Similar to the TRs, each BS uses a group generator to generate an RBs assignment sequence to the WTs directly connected to it. Upon entering the service domain of the TR, a WT $k_{1}$ is assigned an $\mathrm{RB} g_{\imath}^{k_{1}}(\bmod P)$. This technique is readily extensible to the case in which the TRs assign multiple RBs to a single WT with high traffic requirements. In particular, such an assignment can be regarded as an assignment of one RB to multiple incoming WTs.

The fact that each primitive root spans the entire set of available RBs implies that the proposed scheme is well suited to situations in which the distribution of WTs is not uniform. This is because, as noted hereinabove, one of the characteristics of the cyclic structured sequences is that all the available RBs contribute as elements of the cyclic assignment sequence. Hence, by following its assignment sequence, each RT can assign any of the available RBs given that this RB has not been already assigned by another RT. This enables the proposed scheme to dynamically adjust its RBs assignment to cope with the cases when users are concentrated at a specific location within the cell.

Using this assignment scheme, when $M$ is strictly less than $\phi(P-1)$, the optimal choice of the primitive roots must ensure that the number of hit occurrences at which one RB is assigned to multiple WTs is minimized. (When $M=\phi(P-1)$ the optimization of primitive roots is trivial; all the primitive roots are assigned to all the TRs.) However, further minimization of the number of hits can be obtained by using the result in Lemma 1. In particular, using this result it can be seen that the number of hits can be minimized, not only over the sequences generated by the primitive roots, but also over cyclically shifted versions thereof. In this case, in addition to a 
primitive root $g_{\imath}$, each TR is assigned a cyclic shift $s_{\imath} \in\{0, \ldots P-2\}$. Using the primitive-root-cyclic-shift (PRCS) pair $\left(g_{\imath}, s_{\imath}\right)$ the $\ell$-th TR assigns the $\mathrm{RB} r_{\ell}$ to its $k_{1}$-th incoming WT, where

$$
\ell=g_{\imath}^{k_{1}+s_{\imath}} \quad(\bmod P)
$$

In Chapter 7 we will show that augmenting the search for sequences to include the cyclic shifts $\left\{s_{\imath}\right\}$ can have a significant impact on the number of hits.

When cyclic shifts are not used in constructing the assignment sequences, the number of hits is minimized over $\left(\begin{array}{c}\phi(P-1) \\ M\end{array}\right)$ primitive roots. However, by introducing the shifts, the number of possible sequences is increased to $(P-1)^{M}\left(\begin{array}{c}\phi(P-1) \\ M\end{array}\right)$. Although relatively large, this number is significantly smaller than $\left(\begin{array}{c}(P-1)^{\prime} \\ M\end{array}\right)$, which is the number of distinct assignment sequences. To appreciate this gain in design complexity, we consider the case of $P=41$ and $M=3$. In this case, the number of sequences generated by the proposed method is $3.548 \times 10^{7}$, whereas the total number of distinct sequences is greater than $9 \times 10^{142}$.

Since for a given number of RBs, the assignment sequences have to be designed once for each $M \leq \phi(P-1)$, the proposed method for designing assignment sequences incurs a negligible long term computational cost.

We will show in Chapter 7 that the proposed method yields favourable autonomous RB assignments. However, these assignments are only optimal with respect to the set of cyclically generated sequences, but not necessarily optimal with respect to the set of all possible sequences. In the following section we will introduce a metric which will enable efficient optimization of PRCS pairs. 


\subsection{A Metric For Selecting PRCS Pairs}

In order to facilitate the selection of the assignment sequences, it is useful to associate with each sequence a metric that reflects the number of hits that result from using this sequence. We begin by associating a $(P-1) \times(P-1)$ load matrix with each TR $i$, a primitive root $g_{2}$ and a cyclic shift $s_{\imath}$. We will denote this matrix by $X\left(g_{\imath}, s_{\imath}\right), i \in\{1, \ldots, M\}$, and $s_{\imath}=0, \ldots, P-2$. The $\left(\ell_{1}, \ell_{2}\right)$-th entry of $X\left(g_{\imath}, s_{\imath}\right)$ is given by

$$
\left[X\left(g_{\imath}, s_{\imath}\right)\right]_{\ell_{1}, \ell_{2}}=\left\{\begin{array}{l}
1 \text { if } \ell_{1} \in\left\{g_{\imath}^{1+s_{\imath}}(\bmod P), \ldots, g_{\imath}^{\ell_{2}+s_{\imath}}(\bmod P)\right\}, \text { and } \\
0 \text { otherwise. }
\end{array}\right.
$$

As an example, suppose that the PRCS pairs $\left(g_{\imath}, s_{l}\right)$ and $\left(g_{\jmath}, s_{\jmath}\right)$ yield the assignment sequences $(1,4,3,2)$ and $(2,3,1,4)$ for the $i$-th and $j$-th TRs, respectively. The corresponding load matrices, $X\left(g_{\imath}, s_{\imath}\right)$ and $X\left(g_{\jmath}, s_{\jmath}\right)$, are

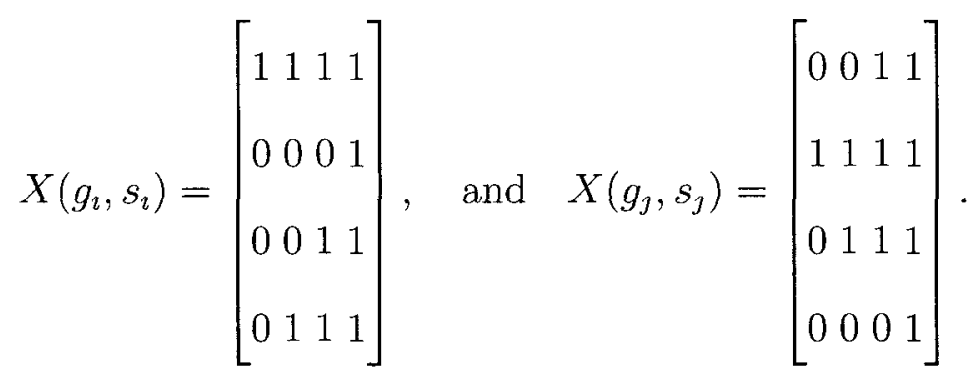

The inner product of the $k_{1}$-th column of $X\left(g_{2}, s_{\imath}\right)$ and the $k_{2}$-th column of $X\left(g_{j}, s_{\jmath}\right)$ yields the number of hits when TRs $i$ and $j$ are loaded by $k_{1}$ and $k_{2}$ WTs, respectively. This inner product is given by the $\left(k_{1}, k_{2}\right)$-th entry of what we call the pairwise hit matrix $H_{\imath, 3}$, where

$$
H_{\imath, \jmath}=X^{T}\left(g_{\imath}, s_{\imath}\right) X\left(g_{\jmath}, s_{\jmath}\right) .
$$


For the hit matrices given in (5.3), the resulting pairwise hit matrix between TRs $\imath$ and $\jmath H_{\imath, \jmath}$ is:

$$
H_{\imath, 3}=\left[\begin{array}{llll}
0 & 0 & 1 & 1 \\
0 & 0 & 1 & 2 \\
0 & 1 & 2 & 3 \\
1 & 2 & 3 & 4
\end{array}\right] .
$$

For instance, from this matrix, the number of hits when the $\imath$-th TR is loaded by 2 WTs and the $\jmath$-th TR is loaded by 3 WTs is given by the entry $\left[H_{\imath, 3}\right]_{2,3}=1$.

Let us denote the load of the $\imath$-th TR by $k_{\imath}, \imath=1, \ldots, M$, and denote the total number of hits observed by the $M$ TRs by $Z\left(k_{1}, \ldots, k_{M}\right)$. Then, it can be seen that

$$
Z\left(k_{1}, \ldots, k_{M}\right)=\sum_{\imath=1}^{M-1} \sum_{\jmath=\imath+1}^{M}\left[H_{\imath, j}\right]_{k_{\imath}, k_{j}} .
$$

This expression is useful to determine the number of hits when the instantaneous load of each TR is known. However, in practical cellular networks the loads of TRs may vary rapidly, which renders timely communication between TRs costly and unrealistic. Furthermore, in the absence of centralized coordination, which is the case consdiered herein, autonomous identification of the loads of all TRs in the cell is not guaranteed to be successful. Thus, to enable a flexible autonomous RB assignment that adapts to bursty traffic and nonuniform WT distribution, the metric for choosing the PRCS pairs must not depend on the instantaneous load. Hence, for the proposed scheme we will choose the PRCS pairs that minimize the average, rather than the instantaneous, number of hits. In this case, the metric for selecting the PRCS pairs can be expressed as

$$
C(K)=\frac{1}{\left(\begin{array}{l}
K-1 \\
M-1
\end{array}\right)} \sum_{\left(k_{1},, k_{M}\right) \in \mathcal{S}_{K}} Z\left(k_{1}, \ldots, k_{M}\right)
$$


where $\mathcal{S}_{K} \triangleq\left\{\left(k_{1}, \ldots, k_{M}\right) \mid \sum_{\imath=1}^{M} k_{\imath}=K, k_{\imath} \in \mathbb{N}, i=1, \ldots, M\right\}$. Hence, $C(K)$ is equal to the number of hits averaged over all possible $M$ partitions of $K$ where all partitions are equiprobable. Note that, since each PRCS pair yields a particular $\mathrm{RB}$ assignment sequence, $C(K)$ is a deterministic quantity that can be computed $a$ prorr independently of the channel realizations. The computation of $C(K)$ can be performed off-line and the optimal PRCS pairs can be stored in a look-up table either at the BS or at the TRs.

\subsubsection{Computational Complexity of $C(K)$}

To assess the computation complexity of evaluating $C(K)$, we note that the entries of the matrices $\left\{X\left(g_{\imath}, s_{2}\right)\right\}$ take on values from $\{0,1\}$. Hence, the cost of the entry-wise multiplications in (5.4) is negligible, and only addition operations contribute to the computational cost of evaluating $C(K)$. In particular, the number of addition operations required for computing each of the $M(M-1) / 2$ matrices $H_{\imath, 3}$ in $(5.4)$ is $(P-1)^{2}(P-2)$ and the number of addition operations required for computing $Z$ in (5.6) is $M(M-1) / 2-1$. To compute the number of addition operations required to compute $C(K)$ in (5.7), we note that the cardinality of the set $\mathcal{S}_{K}$ is equal to the number of ways an integer can be partitioned into $M$ ordered parts. This number is given by $\left(\begin{array}{l}K-1 \\ M-1\end{array}\right)$ [25]. Hence, the total number of addition operations required to compute $C(K)$ is bounded by

$$
\frac{1}{2} M(M-1)\left((P-1)^{2}(P-2)+\left(\begin{array}{l}
K-1 \\
M-1
\end{array}\right)\right) .
$$

From (5.8), it can be seen that computing $C(K)$ involves a reasonable number of additions for small-to-moderate values of $K$ and $M$. However, because of the last term, $C(K)$ becomes computationally prohibitive when $K$ and $M$ are large. 


\subsubsection{Sequence Selection}

When the system load, $K$, is known a prior, the metric $C(K)$ provides an efficient means for assessing the number of hits resulting from choosing a particular set of PRCS pairs for generating the assignment sequences of the TRs. However, when the system load is not known, an alternative is to select one set of PRCS pairs to be used with all possible system loads. Such a set can be chosen to minimize the average of $C(K)$ over all $K \in\{1, \ldots, N\}$, which is proportional to $\sum_{K=1}^{N} C(K)$. In

Chapter 7 we will show that the set of PRCS pairs that minimizes $\frac{1}{N} \sum_{K=1}^{N} C(K)$ yields a performance close to that yielded by the set of PRCS pairs that minimize $C(K)$ at each $K$. In the next section, we will propose a technique by which the number of hits can be reduced. This technique relies on incorporating additional information to which the TRs may have access. Even though in this case the metric $\sum_{K=1}^{N} C(K)$ only represents an upper bound on the number of hits, in Chapter 7 we will show that the sequences chosen according to this metric still yield favourable performance.

\subsection{Further Enhancement of the Resource Block Assignment Scheme}

In the previous section, we provided an efficient scheme for designing RB assignment sequences for TRs in the absence of CQIs. In this scheme, a set of PRCS pairs is selected to ensure that the sequences generated by (5.1) yield a small number of hits. In this section, we will exploit the deterministic nature of the proposed assignment sequences to further reduce the number of hits. In particular, we will show that, using these sequences, the TRs will be able to identify the sequence with which a hit has occurred, to infer the loads of other TRs, and to subsequently utilize this infor- 
mation to avoid future hits. To do this, we assume that the $i$-th TR is aware of the PRCS pairs $\left\{\left(g_{\jmath}, s_{\jmath}\right)\right\}_{\jmath=1, \jmath \neq \imath}^{M}$, of the other $M-1$ TRs in addition to its prescribed $\left(g_{\imath}, s_{2}\right)$-pair. This information about the PRCS pairs of neighboring relays can be injected by the BS in the first communication between the TR and the BS. This has to be done once, when the WT first announces to the BS that it will serve as a TR. Hence, the associated overhead of this process can be considered negligible and does not affect the autonomous nature of the proposed scheme. For randomly generated sequences, the TRs do not have information about the RB assignments of other TRs and hence will not be able to avoid future hits using the technique described below.

\subsubsection{Hit Source Identification and Avoidance (HIA)}

With the set of PRCS pairs $\left\{\left(g_{\imath}, s_{\imath}\right)\right\}_{\imath=1}^{M}$ available, each TR can generate the assignment sequences used by other TRs. When a TR detects a hit, it examines the sequences of the other TRs. First, it determines the RBs on which no hits were detected in the past and subsequently eliminates some of the TRs from the set of potential hit sources. However this information may not be sufficient to determine the exact sequence with which a hit has occurred. In order to resolve this ambiguity, the TR waits for more hits to occur. Incorporating this information, the TR is able to reduce the number of elements in its ambiguity sets. We will refer to this procedure as the hit identification and avoidance (HIA) algorithm, which can be summarized in the following steps:

- A TR identifies a hit on a certain RB, say RB.

- The TR compares its previously assigned RBs with the entries preceding $\hat{R B}$ in the assignment sequences of the other TRs. 
- Using this comparison, the HIA excludes a subset of TRs from the set of potential hit sources.

- If the hit source is identified, the HIA algorithm avoids future hits with this source.

- If the hit source is not identified, the TR continues to assign the RBs according to its sequence until another hit occurs, and the algorithm is repeated.

Hence, it can be seen that the operation of the HIA algorithm depends solely on the current loads of the TRs, but not on the instants at which the WTs access the system. Furthermore, it can be verified that the operation of the HIA algorithm is not affected if some TRs fail to identify the hit source. This is because, in identifying the hit source, the HIA algorithm depends on the RBs that are assigned without hits. However, if a TR does not identify a hit source, it assigns RBs that are already assigned by other TRs. Being already assigned, those RBs are discarded by the HIA algorithm of other TRs.

The HIA procedure is performed by each TR and can be represented by the flow chart in Figure 5.1. In this figure we use $\mathcal{R}_{a}$ to denote the set that contains the RBs already assigned by TR $i$. When TR $i$ detects a hit on a certain RB, we associate with each TR $m \neq i$ a set $\mathcal{R}_{m}$ which contains the RBs preceding the one at which the hit has occurred. In the flow chart we use $\overline{\mathcal{R}} i$ to denote the ambiguity set, which, for the $i$-th TR, is initialized with the set $\{m\}_{m=1, m \neq i}^{M}$. Upon identification, the index of the hit source is denoted by $m_{s}$.

We now provide a three TR example to illustrate the operation of the HIA algorithm. 


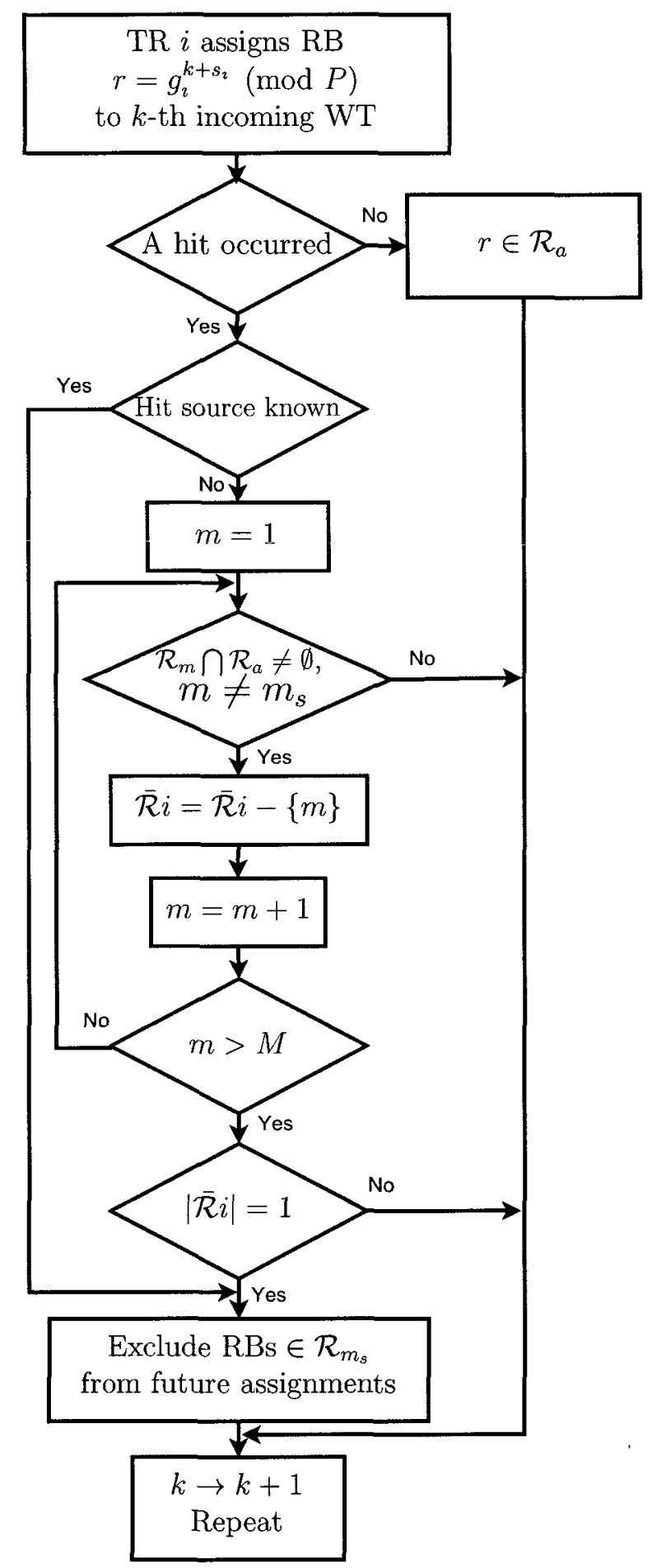

Figure 5.1: Flow chart of the hit identification and avoidance (HIA) technique. 
Example 1. Let $M=3, P=17, s_{\imath}=0, i=1,2,3$, and $g_{1}=3, g_{2}=5$ and $g_{3}=6$, where $g_{1}, g_{2}$ and $g_{3}$ belong to the set of primitive roots of 17 . The sequences generated by $(5.1)$ with $\left\{\left(g_{\imath}, s_{\imath}\right)\right\}_{\imath=1}^{3}$ are given in the following table.

\begin{tabular}{c|cccccccccccccccc}
$\left(g_{1}, s_{1}\right)$ & 3 & 9 & 10 & 13 & 5 & 15 & 11 & 16 & 14 & 8 & 7 & 4 & 12 & 2 & 6 & 1 \\
\hline$\left(g_{2}, s_{2}\right)$ & 5 & 8 & 6 & 13 & 14 & 2 & 10 & 16 & 12 & 9 & 11 & 4 & 3 & 15 & 7 & 1 \\
\hline$\left(g_{3}, s_{3}\right)$ & 6 & 2 & 12 & 4 & 7 & 8 & 14 & 16 & 11 & 15 & 5 & 13 & 10 & 9 & 3 & 1
\end{tabular}

To illustrate the HIA procedure, we consider the case in which the loads of the three TRs are given by $k_{1}=4, k_{2}=3$ and $k_{3}=0$. First, TR 2 assigns RB 6 and no hit is detected. Since this RB is the first to be used by TR 3, TR 2 realizes that $k_{3}=0$. Now, suppose that $k_{2} \rightarrow k_{2}+1$ and, based on its sequence, TR 2 assigns RB 13 to the incoming WT. Since this RB is already assigned by TR 1, both TRs detect a hit, and TR 2 identifies TR 1 as the hit source. However, because the RBs 3,9 and 10 are not used by TRs 2 and 3 , TR 1 is unable to determine the TR with which the hit at RB 13 has occurred. Suppose that $k_{1} \rightarrow k_{1}+1$ and TR 1 assigns RB 5 to the incoming WT. TR 1 detects a hit, but is still unable to determine the hit source. This is because RB 5 is not preceded by any of the successfully assigned RBs in both TR 2 and TR 3 . When $k_{1} \rightarrow k_{1}+1$, TR 1 assigns RB 15 to the incoming WT and no hit is detected. Since TR 3 assigns RB 15 before RB 13 and no hit was detected at RB 15, TR 1 concludes that the hit source was TR 2.

With the hit source identified, a TR is able to determine a subset of the RBs that are already used by other TRs. Hence, to avoid definite hits, the TR must skip these RBs in future assignments. For instance, in the previous example, after identifying TR 1 as the hit source at $k_{1}=k_{2}=4$, TR 2 must avoid assigning RBs 3,9 and 10 . 
For further illustration we provide another example of the HIA algorithm:

Example 2. Let $M=3, P=19, s_{\imath}=0, i=1,2,3$, and $g_{1}=2, g_{2}=10$ and $g_{3}=13$. The sequences generated by (5.1) with $\left\{\left(g_{\imath}, s_{\imath}\right)\right\}_{\imath=1}^{3}$ are given in the following table.

\begin{tabular}{c|cccccccccccccccccc}
$\left(g_{1}, s_{1}\right)$ & 2 & 4 & 8 & 16 & 13 & 7 & 14 & 9 & 18 & 17 & 15 & 11 & 3 & 6 & 12 & 5 & 10 & 1 \\
\hline$\left(g_{2}, s_{2}\right)$ & 3 & 9 & 8 & 5 & 15 & 7 & 2 & 6 & 18 & 16 & 10 & 11 & 14 & 4 & 12 & 17 & 13 & 1 \\
\hline$\left(g_{3}, s_{3}\right)$ & 10 & 5 & 12 & 6 & 3 & 11 & 15 & 17 & 18 & 9 & 14 & 7 & 13 & 16 & 8 & 4 & 2 & 1
\end{tabular}

Consider the case where the loads of the three TRs $k_{1}, k_{2}$ and $k_{3}$ are 2,3 and 4 , respectively. At this point, no hits are detected among any of the TRs. Now let us assume that another WT enters the premises of TR 1 and hence $k_{1} \rightarrow k_{1}+1$. In this case TR 1 and TR 2 detect the occurrence of a hit. TR 2 checks the sequences of TR 1 and TR 3 and concludes that the hit source is TR 1. This is because, had the hit source been TR 3, all the RBs preceding RB 8 in the sequence of TR 3 would have been assigned. Hence a hit should have been detected when assigning RB 3 and RB 9 which did not happen. With the hit source identified TR 2 avoids assigning RB 2 and RB 4 to avoid definite hits. Similarly TR 1 tries to identify the hit source given that RB 2 and RB 4 are already assigned. However it fails to identify the hit source as both RBs come after RB 8 in both the sequences of TR 2 and TR 3 .

In some scenarios it may be possible for the TRs to identify the hit sources immediately by their modulation schemes. In these cases, avoidance becomes more effective in reducing the number of hits, as will be shown in Chapter 7. We refer to such cases where the hit source is identified immediately as immediate HIA. To 
illustrate, the mechanism of the proposed assignment scheme given that immediate HIA is employed we present the following example.

Example 3. Consider the case where the loads of the three TRs $k_{1}, k_{2}$ and $k_{3}$ are 2,3 and 2 , respectively and the number of available $\mathrm{RBs}, N=16$. Let $s_{\imath}=0, i=1,2,3$, and $g_{1}=3, g_{2}=6$ and $g_{3}=10$. The sequences generated by (5.1) with $\left\{\left(g_{\imath}, s_{\imath}\right)\right\}_{\imath=1}^{3}$ can be represented by the following table.

\begin{tabular}{c|cccccccccccccccc}
$\left(g_{1}, s_{1}\right)$ & 3 & 9 & 10 & 13 & 5 & 15 & 11 & 16 & 14 & 8 & 7 & 4 & 12 & 2 & 6 & 1 \\
\hline$\left(g_{2}, s_{2}\right)$ & 6 & 2 & 12 & 4 & 7 & 8 & 14 & 16 & 11 & 15 & 5 & 13 & 10 & 9 & 3 & 1 \\
\hline$\left(g_{3}, s_{3}\right)$ & 10 & 15 & 14 & 4 & 6 & 9 & 5 & 16 & 7 & 2 & 3 & 13 & 11 & 8 & 12 & 1
\end{tabular}

Given the previous load levels, TR 1 will assign RBs 3 and 9, TR 2 will assign RBs 6, 2 and 12 and TR 3 will assign RBs 10 and 15. To illustrate our scheme, let us assume that $k_{1} \rightarrow k_{1}+1$, resulting in the occurrence of a hit between TR 1 and TR 3 . Since immediate HIA is employed, TR 1 identifies TR 3 as the hit sources however it is not able to avoid any hits as RB 10 is the first in the cyclic sequence adopted by TR 3 . On the other hand TR 3 identifies TR 1 as the hit source and accordingly it avoids assigning RBs 3 and 9. This is because, given that a hit occurred with TR 1 at RB 10 and that TRs follow the cyclic sequences in assigning their RBs, TR 3 can infer that RBs 3 and 9 are already assigned by TR 1 to other WTs. As for hit resolution, since TR 3 was the first to assign RB 10, it retains it while TR 1 assigns to its incoming WT the following RB in its sequence which is RB 13.

In our scheme, we considered the case in which the system load only increases according to a counting process as we illustrated in Chapter 4. However our proposed scheme is still extensible to the case where we have dynamic system loads. In this case, 
When a WT exits the premises of a TR, the WT releases its assigned RB. The TR then releases the last chronically assigned $\mathrm{RB}$ and switch the WT employing it to the RB that was released by the exiting WT. This is because the RBs at the beginning of the cyclic assignment sequence are most likely to observe less hits. Moreover, when a TR reaches the end of its assignment sequence, and still has incoming WTs, it generates a sequence of the avoided RBs and follow the same procedure described hereinabove in allocating the RBs to these WTs. We note that in the second phase of the assignment where the sequences consists only of the avoided RBs, the HIA algorithm can still be employed to minimize the number of hit occurrences.

\subsubsection{Computational Complexity of the HIA Algorithm}

First we note that the HIA algorithm does not involve any multiplications, and its computational complexity arises only from the comparison of the assignment sequences when a hit is detected. Each sequence comparison requires at most $P-1$ subtractions. Since the number of TRs is less than or equal to $\phi(P-1)$, for each hit occurrence, the overall complexity of the HIA algorithm is bounded by $(P-1) \phi(P-1) \ll(P-1)^{2}$ subtractions. Hence, the computational complexity of the HIA algorithm is upper bounded by $(P-1)^{2}$ subtraction operations, which can be easily accommodated by standard wireless devices. 


\section{Chapter 6}

\section{Insights In Sequences Selection}

In this chapter, we provide some insights for improving the selection process of the RBs assignment sequences. We start by introducing the effect of employing group isomorphism (discussed in Chapter 3) on the proposed RBs assignment scheme. In the following section, we then exploit some basic concepts of graph theory for efficient selection of the PRCS pairs.

\subsection{Group Isomorphism and Proposed Scheme}

In Chapter 5, we introduced our proposed autonomous RBs assignment scheme. In presenting our scheme, we adopted the multiplicative cyclic group structure introduced in Chapter 3. As illustrated, the core of the proposed scheme is the assignment sequences generated by the PRCS pairs which are exploited by the TRs in assigning their RBs. An important question to be asked is whether this work is extensible to other cyclic group structures such as the additive cyclic groups or not? In other words, will the performance of the proposed RBs assignment scheme be affected by changing the selected cyclic group structure?

The answer to this question would be that there is no need to explore other cyclic group structures. This is because, as illustrated in Chapter 3, all cyclic groups are isomorphic to the additive group $\mathcal{Z}_{n}=\{0,1,2, \ldots, n-1\}$. This indicates that after 
the isomorphic mapping of elements, illustrated in Figure 6.1, all cyclic groups will have the same algebraic structure and properties. In particular, group isomorphism can be considered as a relabeling function and hence all cyclic groups cannot be distinguished from an algebraic perspective.

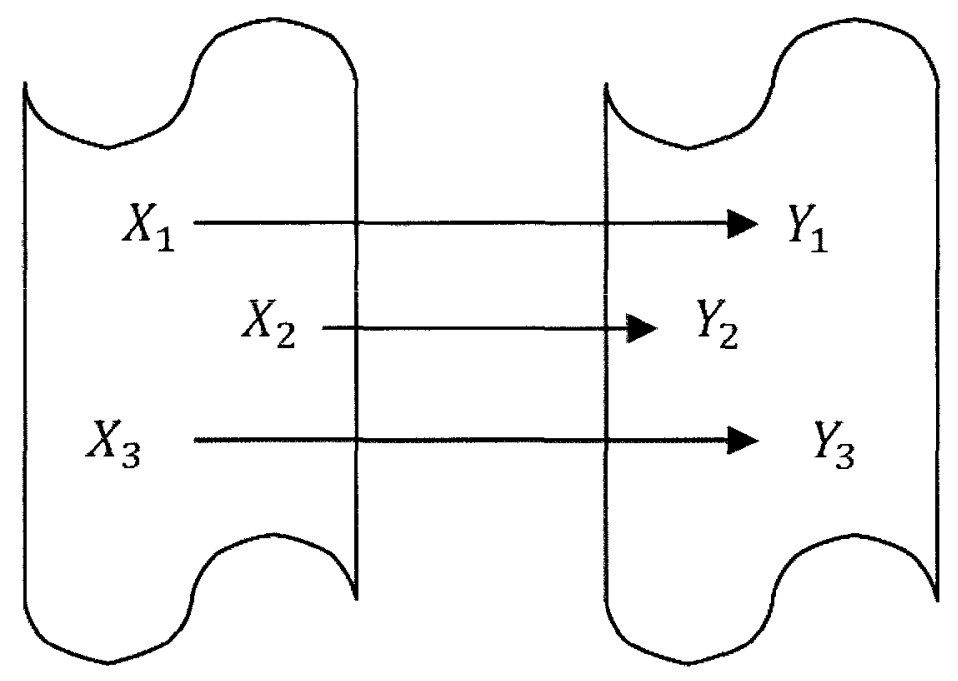

Figure 6.1: One to one mapping between two isomorphic groups.

Accordingly, we summarize this research point by the following statement which broadens the scope of our proposed scheme:

Let $G_{1}$ and $G_{2}$ be two distinct cyclic groups of the same order. Then, for each sequence generated by the group generator $g_{1} \in G_{1}$ there is an identical sequence generated by the group generator $g_{2} \in G_{2}$ under the isomorphic mapping function.

Therefore, by referring to both, the previous conclusion and Lemma 2, we can expect to have sequences that are identical to the sequences generated by the multiplicative cyclic group generators even if a different cyclic group structure is employed as long as the group order is the same. Hence, all cyclic groups, regardless of the adopted group operation, will provide the same performance since the resulting num- 
ber of hit occurrences is a direct consequence of the RBs assignment sequences that are adopted by the TRs.

\subsection{Graph Theory and Sequences Selection}

In our proposed work, the process of sequences selection (PRCS pairs selection) is performed once prior to the startup of the system. However, the number of possible combinations of sequences is directly proportional to both the number of TRs and the number of RBs. Hence an increase in any of these numbers raises the time required for selecting the best performing PRCS pairs that will be adopted by the TRs. In order to decrease the complexity of this process, in Chapter 5, we devised a sequences selection metric. In the same chapter we also formulated the computational complexity associated with the sequences selection metric.

As an extension of our work, we explored the possibility of increasing the speed of the PRCS pairs selection process. In particular, we investigated the graphical representations of cyclic groups as potential means for providing an alternative graphical solution for the selection process.

From graph theory, the graphical notation of a cyclic group is a circle that connects all the elements of the group, as illustrated in Figure 6.2, whereby the elements of the group are located on the circumference of the circle. This circle of elements can be constructed from any of the sequences generated by the group generators of the cyclic group. This is because, as illustrated in Chapter 3, any cyclically generated sequence consists of all the elements of the group. This sequence and its inverse will be referred to as the cyclic circle basis. In this graphical representation, each two adjacent elements in the sequence will be connected.

In this graphical notation, each of the remaining group generators (the ones 


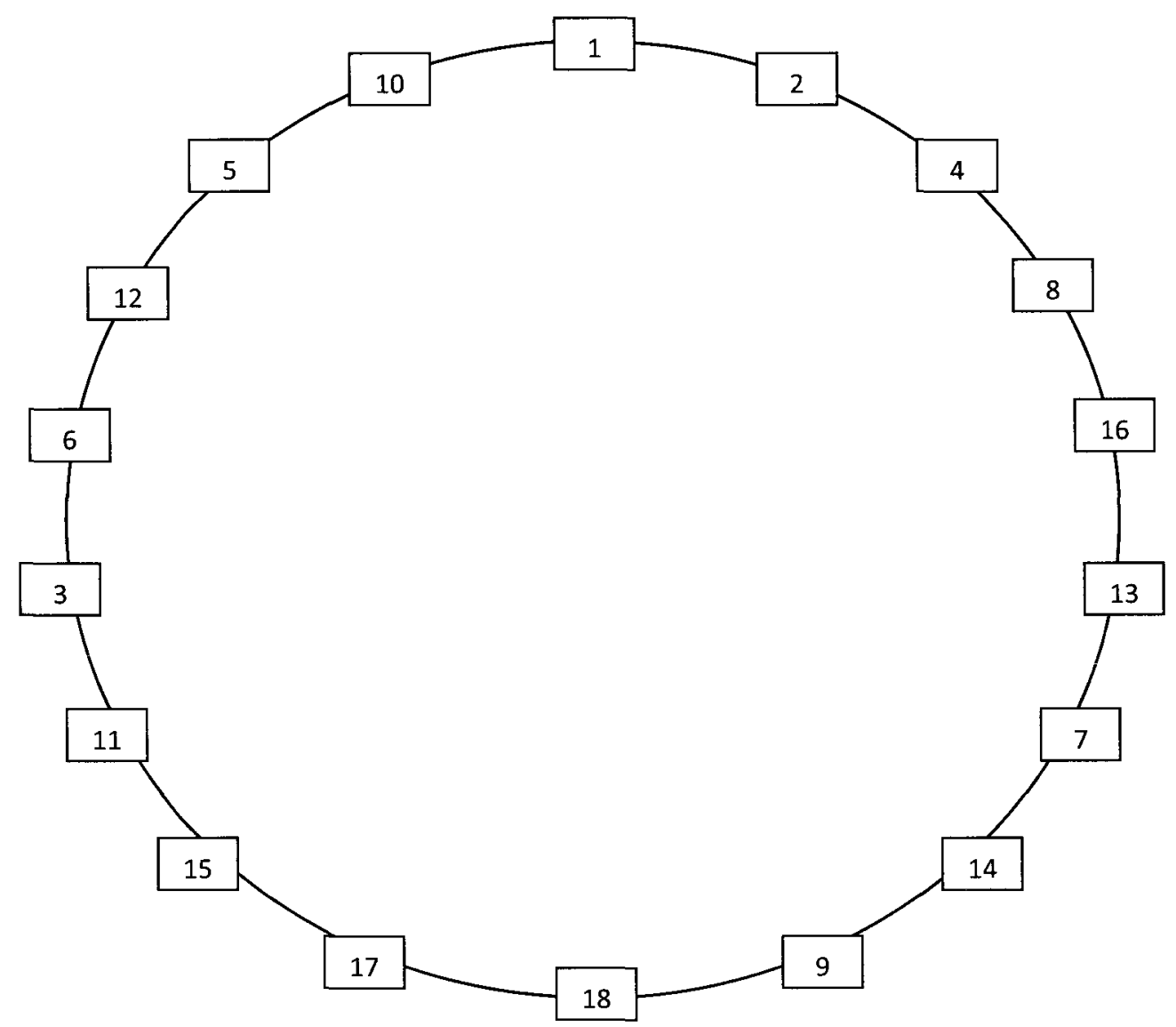

Figure 6.2: A graphical representation of a cyclic group.

that are not used to build the cyclic graph) will result in a unique pattern that connects all the elements of the group. In other words, one cyclically generated sequence and its inverse will be used to construct the circle while each of the remaining cyclically generated sequences and its inverse will contribute to the cyclic graph by a unique pattern that connects all the elements of the group. In this pattern, the lines attributing to the formation of this pattern come from connecting the adjacent elements in the cyclic sequence.We also note that the graphical representation of cyclic groups is unique and depends only on the order of the group. This means that even if a different cyclically generated sequence was used to build the circle, 


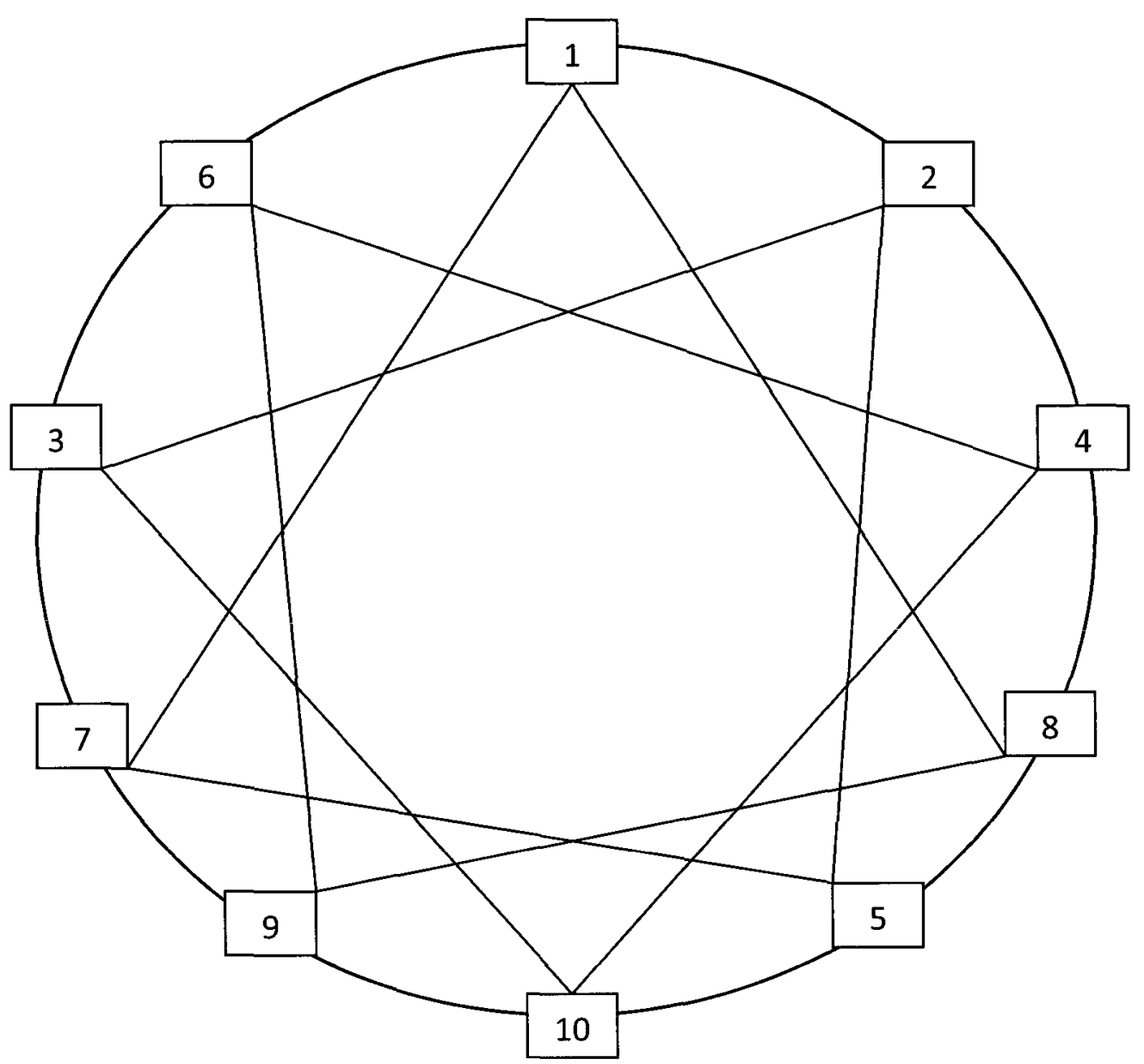

Figure 6.3: A graphical representation of the cyclic group of order 10 including all its group generators' patterns.

the graphical representation of the cyclic group will not be affected. For example, if we consider the multiplicative cyclic group of order 10, the corresponding graphical representation will be as in Figure 6.3:

In this graphical notation, when a cyclic shift is applied to the group generator, its corresponding pattern turns accordingly in either a clockwise or an anti-clockwise direction depending on whether the added cyclic shift was positive or negative. The effect of the cyclic shift is illustrated in Figure 6.4. 


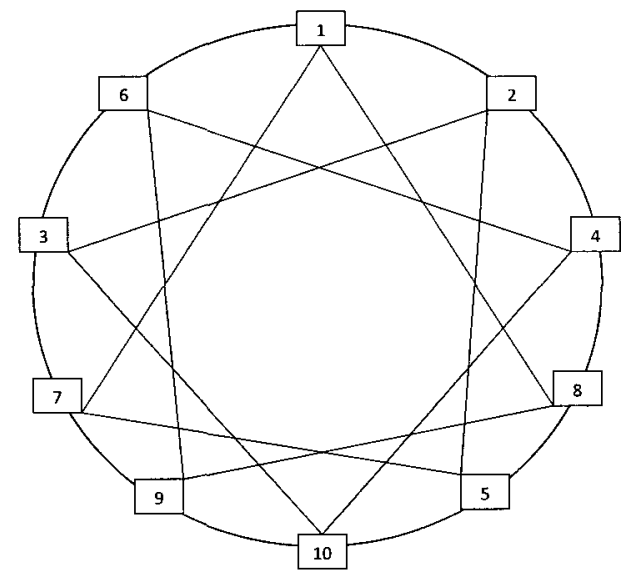

(a)

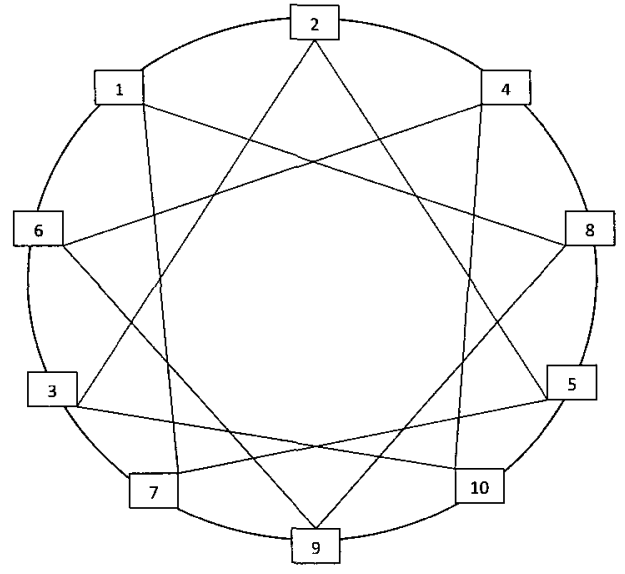

(b)

Figure 6.4: a.Cyclic group of order 10 without cyclic shift.

b.Cyclic group of order 10 with an anti-clockwise cyclic shift of a step size one $(\mathrm{s}=-1)$.

For any cyclic group $G$ of order $n$, The group generators can be divided into pairs. In particular, for each group generator $g_{1} \in G$ there exists a compliment group generator $g_{2} \in G$ which is the arithmetic modulo inverse of the group generator $g_{1}$. This group generator, $g_{2}$, will generate a sequence that is identical to the one generated by $g_{1}$ but in a reverse order. This means that, from a graphical point of view, $g_{1}$ and $g_{2}$ will generate a pattern and its mirror image respectively. For example, if we consider the multiplicative cyclic group of order $n=P-1=16$, one of the group generators of the cyclic group will be $g=3$. Accordingly, the arithmetic inverse of $g$ modulo $P$ will be equal to $g^{-1}=6$. The sequences generated by the two group generators in this example are presented in Figure 6.5.

As an illustrative example, we can solve the trivial two relay case given the previously illustrated graphical tools. In this case, any pair of group generators that consists of a group generator and its inverse can represent a valid solution for the 


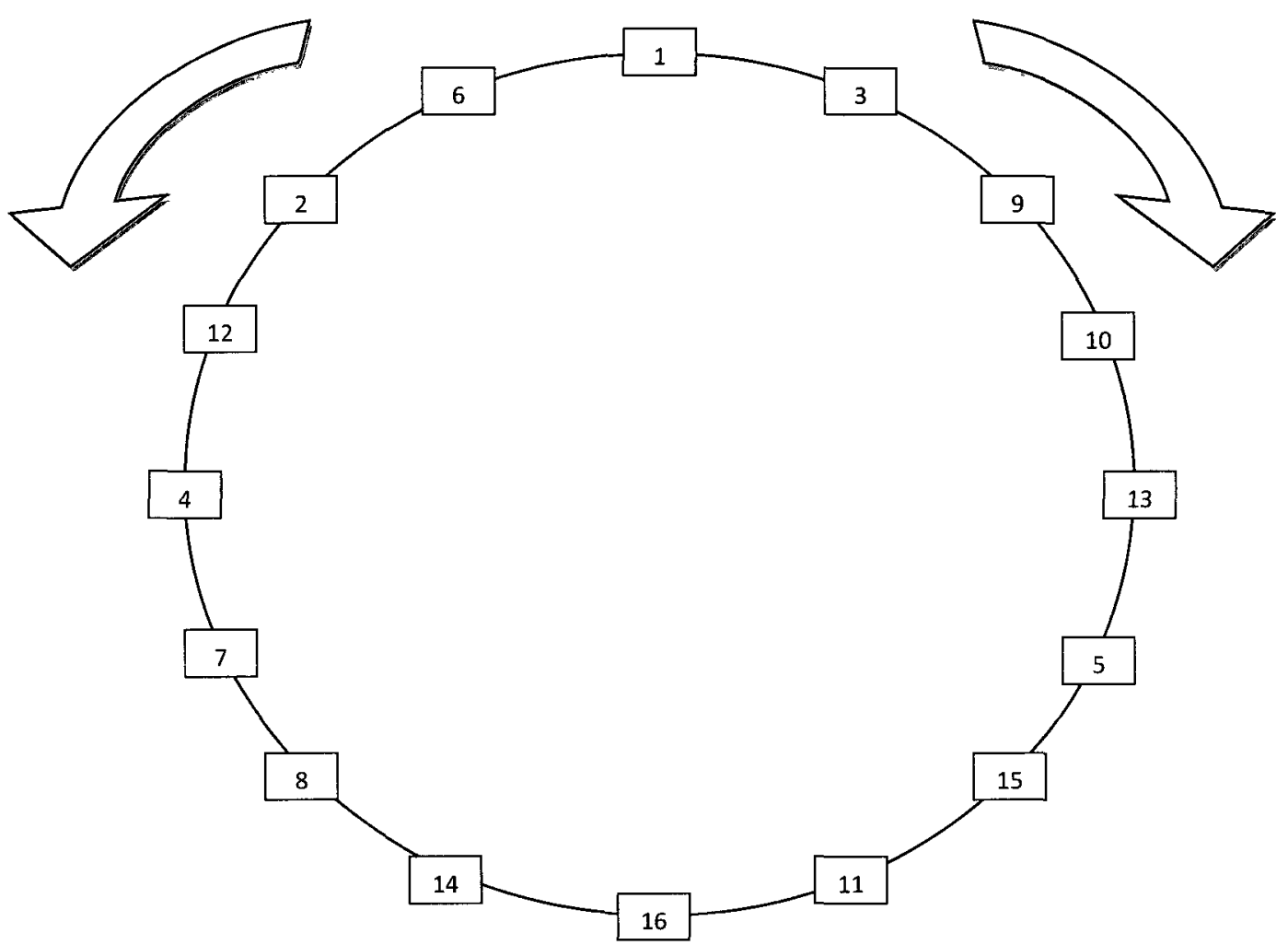

Figure 6.5: A group generator and its inverse of a cyclic multiplicative group of order $\mathrm{n}=\mathrm{P}-1=16$.

problem. The graphical representation of cyclic groups described hereinabove can also be utilized as an alternative for the metric described in Chapter 5. As we will show in the following section, this alternative solution can significantly reduce the computational complexity associated with the PRCS pairs selection process especially in cases where we have large numbers of RBs. The proposed graphical selection technique is presented in the following section.

\subsubsection{A Graphical Selection of PRCS Pairs}

The graphical PRCS selection technique is one of the promising candidates for increasing the speed of the PRCS pairs selection process. This technique can be 
realized by utilizing the above mentioned graphical properties of cyclic groups. To illustrate the proposed graphical selection process, let us consider the simple case where we have $M=3$ TRs and $N=18$ RBs. In this example, we adopt the multiplicative cyclic group structure. Hence, the corresponding group generator pairs will be $\mathcal{U}=\{(2,10),(3,13),(14,15)\}$ where each pair consists of a group generator and its inverse. As noted in the previous section, the graphical notation of any cyclic group is unique as long as the group order is maintained and does not depend on the sequences that are chosen to be the circle basis. Hence, any of the pairs in $\mathcal{U}$ can be chosen to be the circle basis. For this example, we will consider the pair $(2,10)$ to be the basis of cyclic graph. In this case, the corresponding graphical notation of the cyclic graph becomes as presented in Figure 6.6:

Similar to the two relay case, we choose the group generators of the first two TRs to be the basis of the circle. This is because, with the assumption that the total number of WTs is smaller than the number of $\mathrm{RBs}(K<N)$, these two group generators will result in the minimum number of hits. We also note that any pair would result in the same performance and hence the selection is not unique. For this example we choose the group generator pair $(2,10)$ where $g_{1}=2$ and $g_{2}=10$. As for the third $\mathrm{TR}$ in this example, we should choose one of the remaining 4 group generators that would result in minimizing the total number of hits. However, given the fact that the group generators come in pairs and that the metric considered is the average number of hits over all possible load combinations, evaluating the performance of one group generator is sufficient to determine the performance of the two group generators in the pair. This is because the group generators within the pair generate a unique pattern and its mirror image. Hence, comparing the performance over all possible load combinations would result in both of the group generators of 


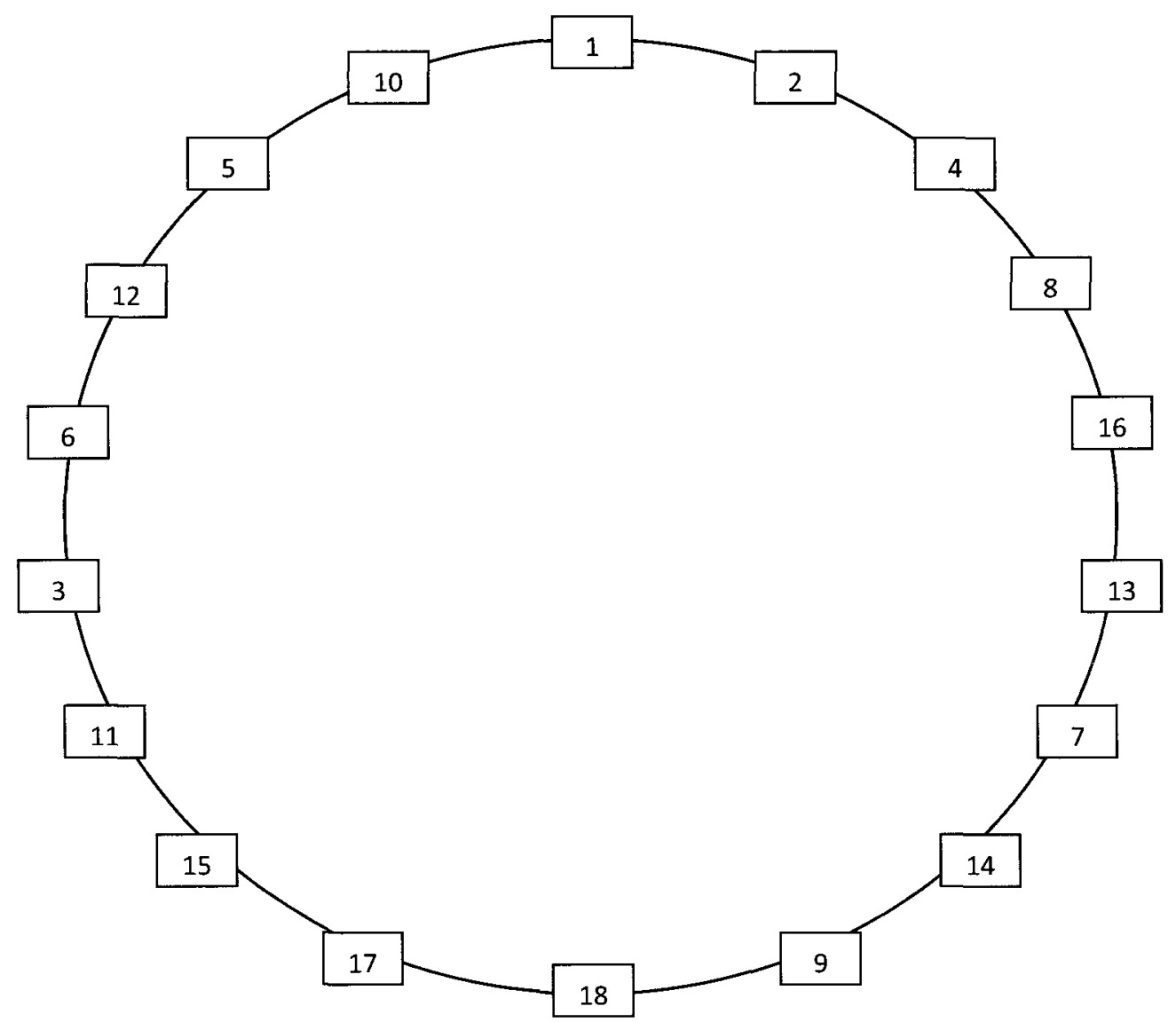

Figure 6.6: The graphical notation of the multiplicative cyclic group of order $18(P=$ 19) with the group generator pair $(2,10)$ being the basis of the circle.

the pair providing identical performance. Therefore, in this example we would have only two group generators to choose from which are 3 and 14 .

We then arbitrary choose one of the group generators and draw the pattern generated by its sequence on the cyclic graph. In this example, we choose $g_{3}=3$ with a cyclic shift $s=0$ and draw its pattern on the cyclic graph as illustrated in Figure 6.7:

We now calculate the number of hits resulting from this selection of group generators over all possible load combinations. This can be done by calculating the number of observed hits between each pair of sequences similar to the pair wise hit matrix $H_{i, j}$ 


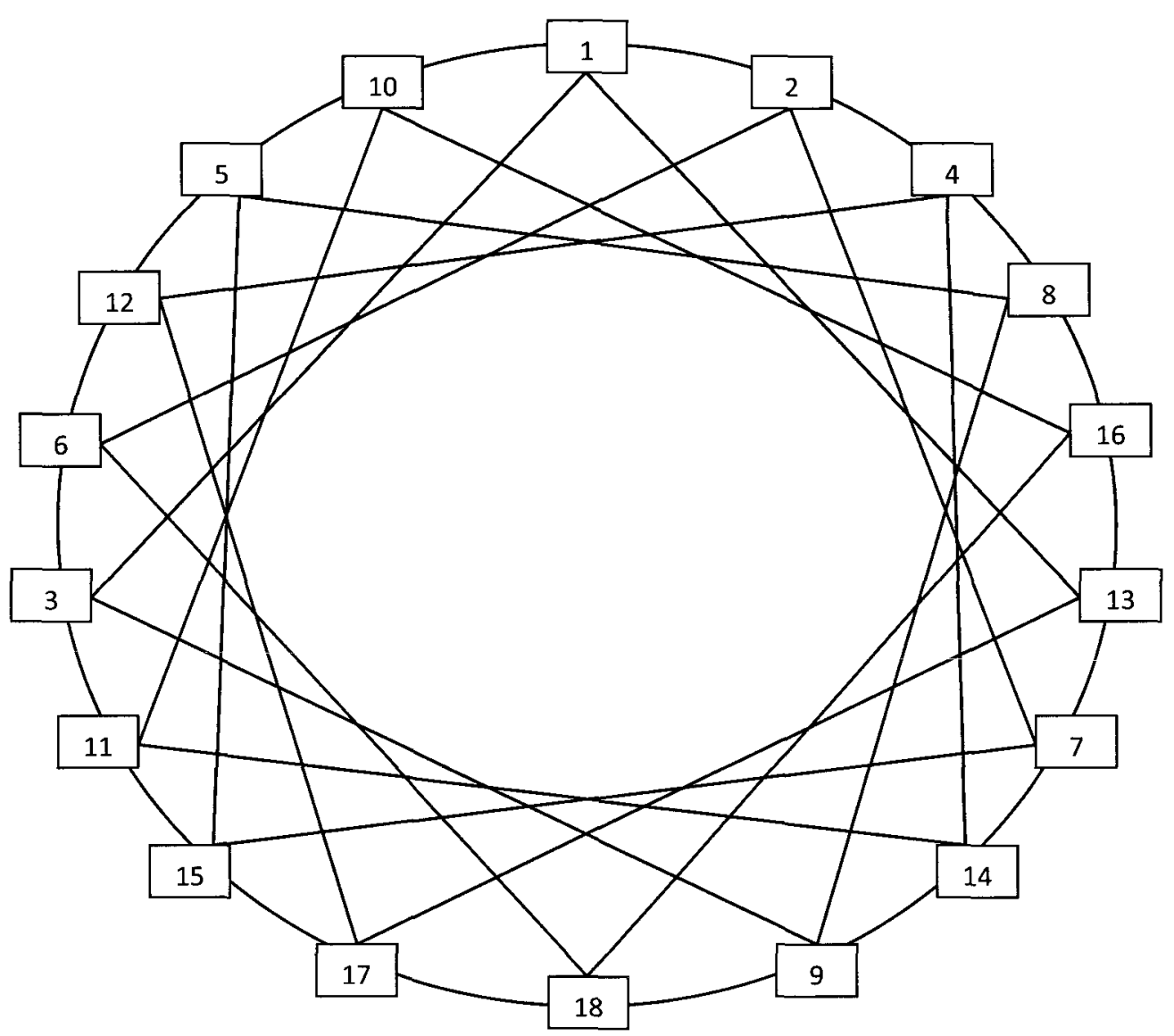

Figure 6.7: The graphical representation of the pattern generated from the group generator 3 in the multiplicative cyclic group of order 18.

in the metric presented in Chapter 5. However since $g_{1}$ and $g_{2}$ come from the same pair where $g_{1}=2$ is the arithmetic inverse of $g_{2}=10$ then no hits will be observed between these two sequences as long as $K<N$. Hence, when calculating the total number of hits observed from this selection, we only consider the corresponding hits between the sequences generated by the pairs $\left(g_{1}, g_{3}\right)$ and $\left(g_{2}, g_{3}\right)$. If we consider the pair $\left(g_{2}, g_{3}\right)$, in order to calculate the total number of hits observed between their sequences we have to add all the resulting hits at each intersection (The occurrence at which the same element appears in both sequences) of the two sequences over all 
possible load combinations. We will refer to the considered intersecting element as the Intersection Element of Interest and denote it by IEI. In other words, since both sequences share the same elements, we have to consider the total number of hits over all the elements of the sequences. For this example, If we consider the pair of sequences generated by the group generators $g_{2}=10$ and $g_{3}=3$, illustrated in the following table, and consider $I E I=3$, then we will notice the occurrence of a hit between the first element in the sequence generated by $g_{3}=3$ and the fifth element in the sequence generated by $g_{2}=10$.

\begin{tabular}{c|cccccccccccccccccc}
$\left(g_{1}, s_{1}\right)$ & 2 & 4 & 8 & 16 & 13 & 7 & 14 & 9 & 18 & 17 & 15 & 11 & 3 & 6 & 12 & 5 & 10 & 1 \\
\hline$\left(g_{2}, s_{2}\right)$ & 10 & 5 & 12 & 6 & 3 & 11 & 15 & 17 & 18 & 9 & 14 & 7 & 13 & 16 & 8 & 4 & 2 & 1 \\
\hline$\left(g_{3}, s_{3}\right)$ & 3 & 9 & 8 & 5 & 15 & 7 & 2 & 6 & 18 & 16 & 10 & 11 & 14 & 4 & 12 & 17 & 13 & 1
\end{tabular}

Given the position of the IEI in both sequences, the resulting number of hits over all possible load combinations due to this element can be calculated by the following formula:

$$
V_{I E I, c_{2}, c_{3}}=\sum_{x_{1}=c_{2}}^{N-c_{3}} \sum_{x_{2}=c_{3}}^{N-x_{1}} \sum_{x_{3}=0}^{N-x_{1}-x_{2}} .
$$

In this formula, the $I E I$ represents the chosen element for which we want to calculate the total number of hits while $N$ represents the number of RBs and $c_{2}$ and $c_{3}$ represent the position of the $I E I$ in the sequences generated by $g_{2}$ and $g_{3}$ respectively. For the previous example, we have $N=18$ and $I E I=3$ while $c_{2}=5$ and $c_{3}=1$. To further illustrate, this value $V_{3,5,1}$ will represent the total number of hits observed over all possible load combinations for $I E I=3$ between the sequences generated by the group generators $g_{2}=10$ and $g_{3}=3$ where the $I E I$ comes in the 
fifth position in the sequence generated by $g_{2}$ and in the first position in the sequence generated by $g_{3} ; c_{2}=5$ and $c_{3}=1$.

We then calculate the total number of hits (denoted by $I_{s e q_{2}, s e q_{3}}$ ) resulting from all intersections between the sequences $s e q_{2}$ and $s e q_{3}$ among all their elements over all possible load combinations according to Equation 6.2 .

$$
I_{s e q_{2}, s e q_{3}}=\sum_{I E I=1}^{N} V_{I E I, c_{2}, c_{3}} .
$$

For this example, $s e q_{2}$ and $s e q_{3}$ represent the sequences generated by the group generators $g_{2}=10$ and $g_{3}=3$. However as noted earlier, to be able to evaluate the performance of the selected group generators we have to consider the total number of hits between the sequences generated by the pairs $\left(g_{1}, g_{3}\right)$ and $\left(g_{2}, g_{3}\right)$. We refer to the total number of hits for a selection of group generators by $I_{\text {total }}$ and evaluate it according to the following equation:

$$
I_{\text {total }}=I_{s e q_{1}, s e q_{3}}+I_{s e q_{2}, s e q_{3}}
$$

In this selection the cyclic shift of all sequences was set to zero; $s_{1}=s_{2}=s_{3}=0$. Hence, after calculating the total number of hits for this selection, we then consider the effect of the additional degree of freedom realized by the cyclic shifts. We start by a cyclic shift $s_{3}=1$ for sequence $\operatorname{seq}_{3}$ then recalculate the total number of hits $I_{\text {total }}$. We note that the cyclic shift is only applied to the sequence that generates the inner pattern and not the cyclic circle basis. In other words only $s_{3}$ is updated while $s_{1}$ and $s_{2}$ are set to zero. The same procedure is repeated for all cyclic shifts $1 \leq s_{3} \leq N-1$. We then choose the cyclic shift that results in the lowest value of $I_{\text {total }}$. The group generator $g_{3}=3$ is then updated to be $g_{3}=14$ and the same procedure is employed to obtain the cyclic shift that provides the best performance. 
We then choose the group generator and the cyclic shift pair that results in the lowest value of $I_{\text {total }}$. The graphical selection of the PRCS pairs can be represented by the flow chart in Figure 6.8:

To conclude, we note that the chosen PRCS pairs will result in the smallest number of hits over all possible load combinations. However, this solution is not unique as selecting a different pair of group generators to be the basis of the cyclic graph will result in a different combination of PRCS pairs with exactly the same number of hits $I_{\text {total }}$.

Although it might be a dilemma to extend this work for larger numbers of TRs due to the difficulty associated with visualizing a higher dimensional graph, the previous procedure can still be extended to the case where we have 4 TRs. In this case, after selecting the PRCS pairs for the 3 TRs scenario, we add to our selected PRCS pairs the arithmetic inverse of the group generator and the negative value of the cyclic shift $\left(g_{3}^{-1},-s_{3}\right)$ which were selected for the third TR. This is because, the inverse of a group generator $g^{-1}$ represents a mirror image of the pattern generated by the group generator $g$. Hence, the cyclic shift has to be mirrored by taking its negative value. We also note that when evaluating $I_{\text {total }}$ for the case where we have 4 TRs, all possible combinations of pairs should be taken into consideration unlike the 3 TRs case where we considered only the pairs $\left(g_{1}, g_{3}\right)$ and $\left(g_{2}, g_{3}\right)$. In this case, we will have to consider the pairs $\mathcal{U}=\left\{\left(g_{1}, g_{2}\right)\left(g_{1}, g_{3}\right),\left(g_{1}, g_{4}\right),\left(g_{2}, g_{3}\right),\left(g_{2}, g_{4}\right),\left(g_{3}, g_{4}\right)\right\}$ where the pair of group generators $\left(g_{1}, g_{2}\right)$ will be the basis of the cyclic circle.

\subsubsection{Computational Complexity of The Graphical Solution}

To assess the computation complexity associated with the graphical selection technique, we note that only addition operations contribute to the computational 


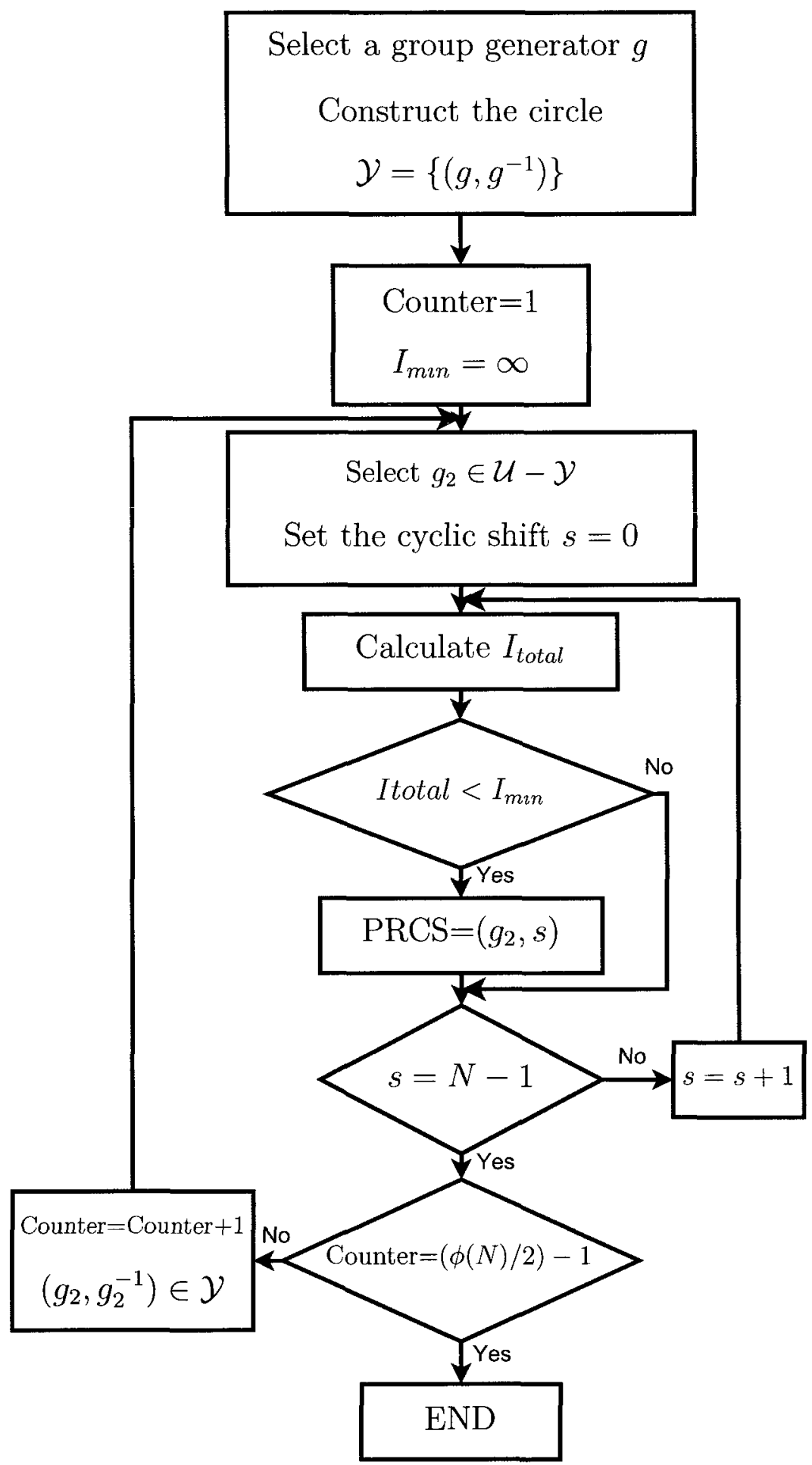

Figure 6.8: The process of the graphical selection of the PRCS pairs. 
cost of evaluating $I_{\text {total }}$. In particular, the number of addition operations required for computing each value of $V_{I E I, c_{1}, c_{2}}$ is bounded by $N^{3}$. In our graphical solution, the PRCS pairs for the first and the second TRs are chosen arbitrary as a group generator and its inverse. Hence, the number of available options of group generators for the third TR can be given by Equation 6.4.

$$
(\phi(N)-2) / 2 .
$$

This is because, as illustrated in the previous section, it is sufficient to test only one of the group generators of each pair. Hence the number of addition operations required to compute $I_{s e q_{1}, s e q_{2}}$ is bounded by the $N^{4}$ as the value $V_{I E I, c_{1}, c_{2}}$ has to be evaluated $N$ times for each value of $I_{s e q_{1}, s e q_{2}}$. Accordingly, from Equation 6.3, the computational complexity for evaluating $I_{\text {total }}$ can be bounded by $(2)\left(N^{4}\right)$.

Based on the proposed graphical technique, the calculation of the value $I_{\text {total }}$ has to be repeated for each cyclic shift and for all the available options of group generators that can be adopted by the third TR. Hence, the computational complexity associated with the graphical PRCS selection technique can be bounded by:

$$
(2)\left(N^{5}\right)(\phi(N)-2) / 2 \text {. }
$$

From 6.5 it can be seen that the computational complexity for selecting the best performing PRCS pairs involves a reasonable number of addition operations even for the cases where we have large number of RBs $N$. We note that in the case where we have 4 TRs instead of 3 , the bound on the computational complexity of the graphical selection technique then becomes:

$$
(((M)(M-1) / 2)-1)\left(N^{5}\right)((\phi(N)-2) / 2) .
$$


This is because in evaluating $I_{\text {total }}$ the number of hits observed between all pairs of group generators (other than the pair $\left(g_{1}, g_{2}\right)$ as it will results in 0 hits when $N<K)$ has to be taken into consideration. 


\section{Chapter 7}

\section{Simulation Results}

In this chapter we consider the performance of the proposed assignment sequences with the HIA scheme and the performance of the random [11], the PNbased, and the optimal centralized schemes at different relative loads $\frac{K}{P-1}$; i.e., the ratio of the currently assigned $\mathrm{RBs}$ to the total number of RBs in the system. For ease of exposition, we restrict our attention to the case in which the number of TRs is $M=3$. For each $K$, the performance is measured by the average number of hits over possible load combinations; i.e., the average overall ordered triples $\left(k_{1}, k_{2}, k_{3}\right) \in\left\{\left(k_{1}, k_{2}, k_{3}\right) \mid \sum_{\imath=1}^{3} k_{\imath}=K, k_{\imath} \geq 0\right\}$. The results of the performance evaluation simulations are presented by the following examples:

Example 4. In this example we consider two cases, one with $P=17$ and the other with $P=41$. For each case, the assignment sequences are generated by (5.1) with the PRCS pairs that minimize $\sum_{K=1}^{P-1} C(K)$; cf. (5.7). For $P=17$, the optimal roots and shifts were found to be: $\left(g_{1}, s_{1}\right)=(3,1),\left(g_{2}, s_{2}\right)=(6,3),\left(g_{3}, s_{3}\right)=(7,10)$, and for $P=41$, they were found to be: $\left(g_{1}, s_{1}\right)=(6,1),\left(g_{2}, s_{2}\right)=(15,2),\left(g_{3}, s_{3}\right)=(19,22)$. Although it may be possible to optimize the choice of the primitive roots and the cyclic shifts for each $K$, our investigations indicate that this approach yields marginal improvement in performance (As illustrated in Example 8) and does not warrant the additional computational complexity. In this example, the TRs do not use the HIA 


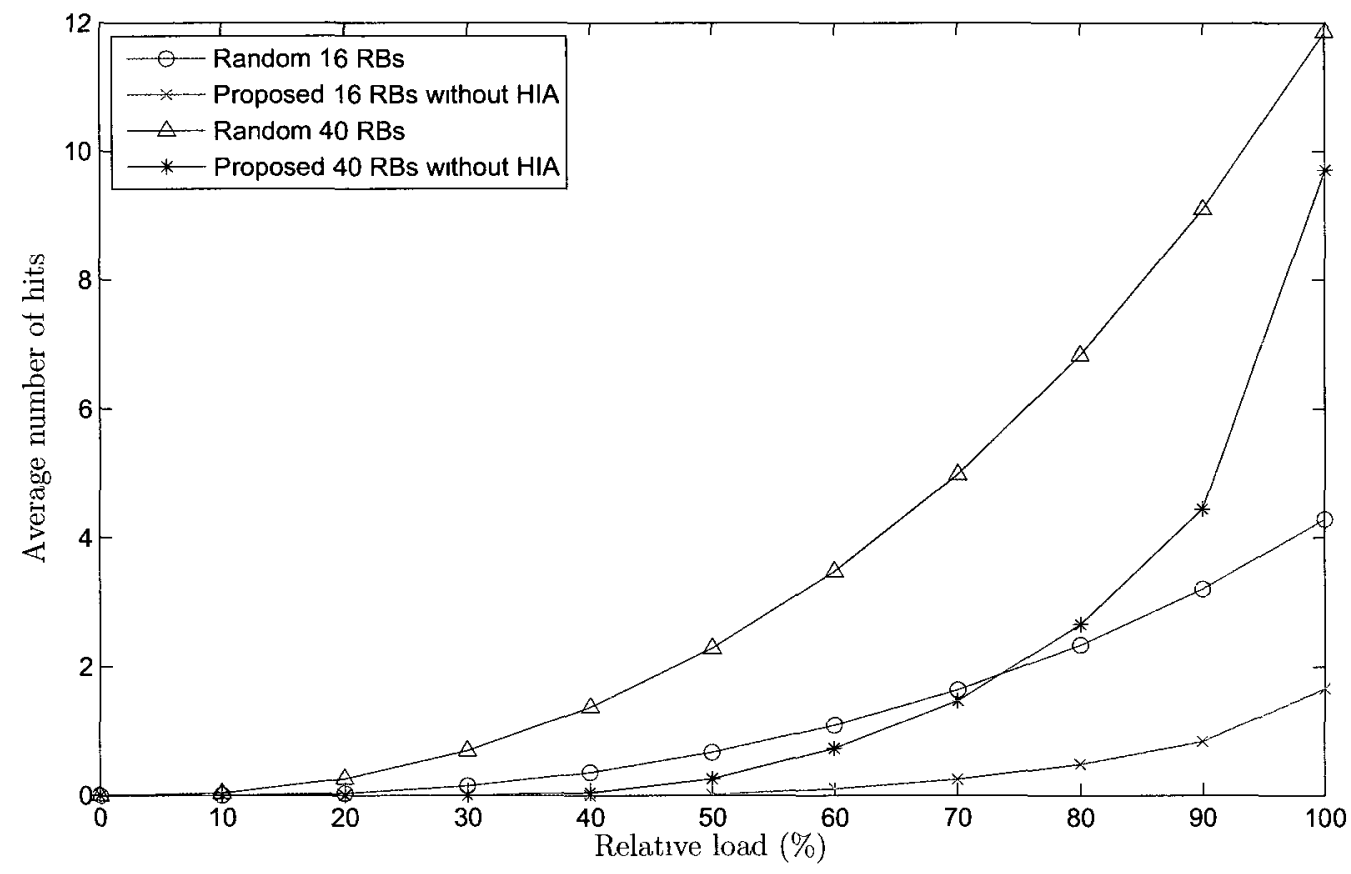

Figure 7.1: Comparison between proposed assignment sequences and the random assignment scheme proposed in [11] for $N=P-1$ RBs with $P=17$ and $P=41$.

technique of Chapter 5. The comparison of the proposed assignment sequences with the random ones proposed in [11] is shown in Figure 7.1.

From this figure, it can be seen that the proposed sequences yield a significant reduction in the average number of hits. For instance, when $\frac{K}{P-1}=90 \%$, the average number of hits produced by the technique in [11] is 3.2 and 9.1 , for $P=17$ and $P=41$, respectively. At the same load, the respective average number of hits produced by the assignment sequences proposed herein is 0.84 and 4.44 .

Example 5. In this example we consider a setup similar to the one in the previous example with $P=41$. However, in this example, the TRs are assumed to know the assignment sequences of other TRs, which enables them to utilize the HIA technique of Chapter 5 . We consider two scenarios, one in which the hit sources are immediately 


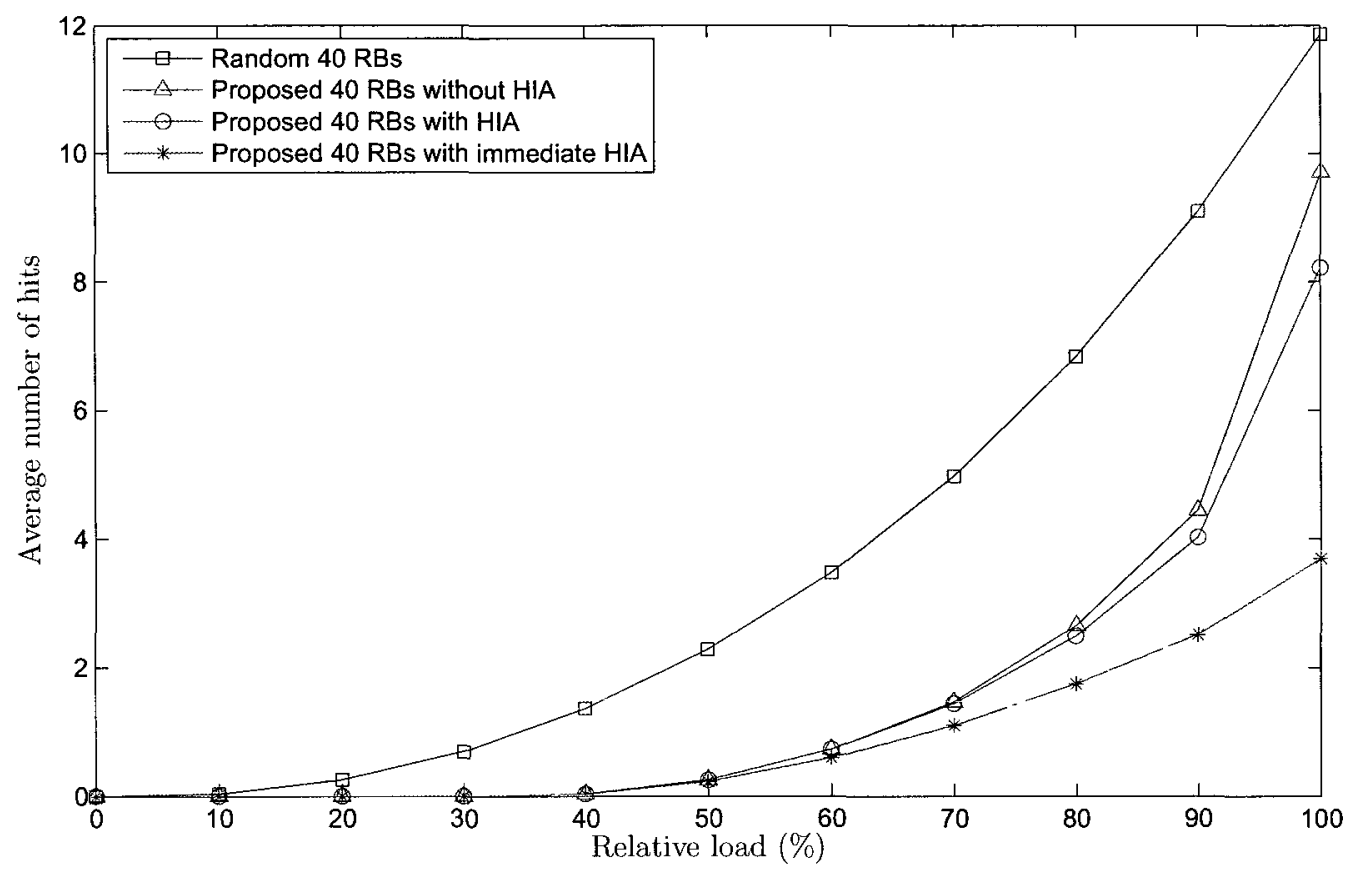

Figure 7.2: Comparison between random assignment scheme proposed in [11] and the proposed assignment for $N=40$ RBs (i.e., $P=41$ ) with and without HIA and with immediate HIA.

identified and another in which the hit sources are identified with the technique of Chapter 5. Notice that the HIA technique of Chapter 5 cannot be applied to the assignment sequences proposed in [11] because of their random structure.

Figure 7.2 demonstrates the impact of using the HIA technique of Chapter 5. For comparison, the average number of hits generated by the random technique is also shown in this figure. When $\frac{K}{P-1}=90 \%$, the average number of hits produced when no hit avoidance is used is 4.44. Using hit avoidance, this number is reduced to 4.03 when the hit source is identified with the HIA technique of Chapter 5 and to 2.5 when the hit source is identified immediately. The average number of hits when the technique in [11] is used is 9.1 .

The metric in (5.7) offers a convenient means for optimizing the primitive roots 


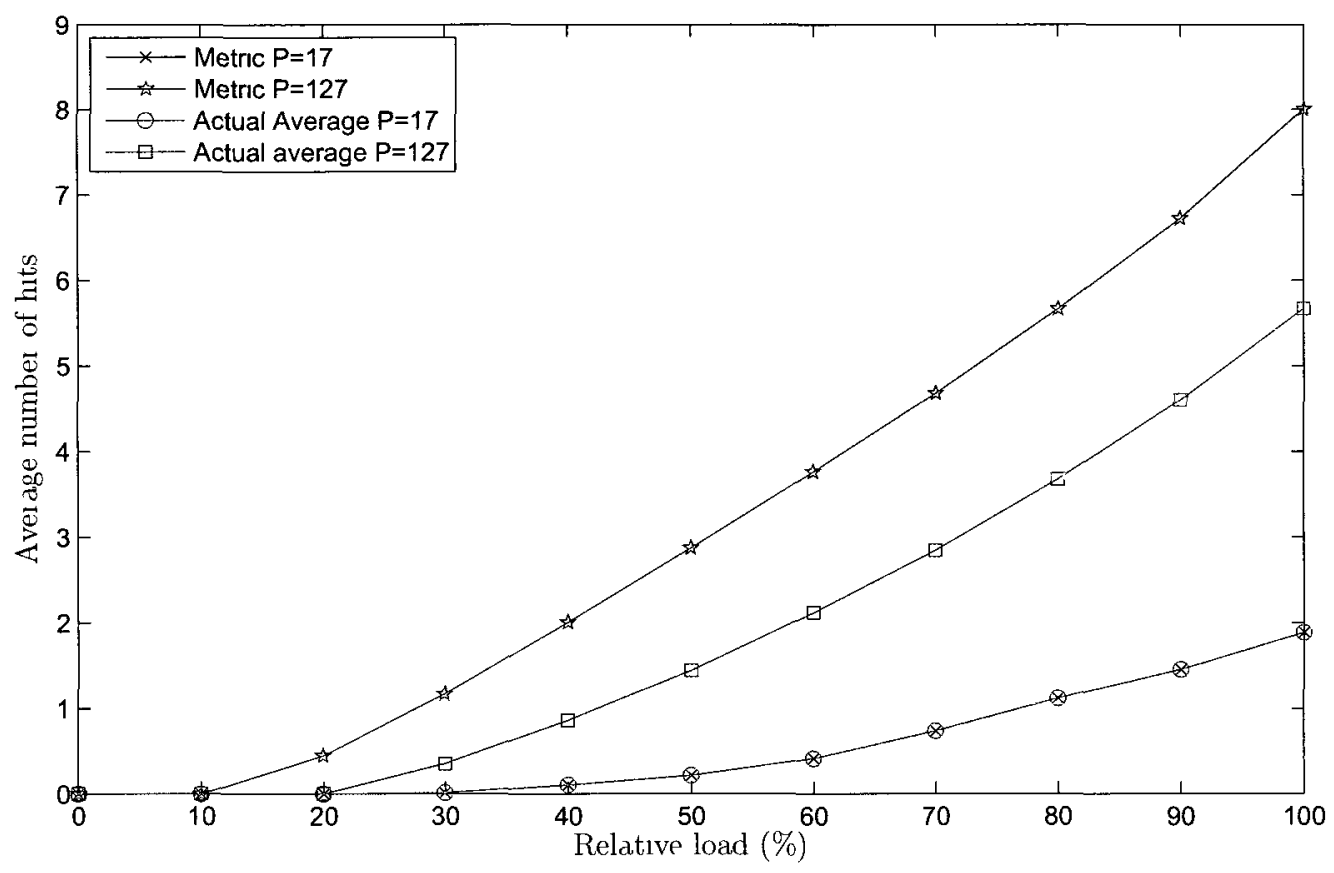

Figure 7.3: Comparison between metric minimizing roots and actual average minimizing roots for $P=17$ and $P=127$ with immediate HIA.

and the cyclic shifts of the assignment sequences. However, this metric does not take the hit source identification and avoidance into consideration. Hence, when the HIA technique is used, the proposed metric yields an upper bound on the actual number of hits.

Example 6. To illustrate the usefulness of the metric, in Figure 7.3 we compare the performance of the proposed scheme when $P=17$ and $P=127$. The cyclic shifts are set equal to zero; i.e., $s_{1}=s_{2}=s_{3}=0$. In all cases the immediate HIA technique is employed, and the primitive roots $\left\{\left(g_{2}\right)\right\}_{t=1}^{3}$ are chosen in two ways: to minimize the metric, which is an upper bound on the average number of hits, and to minimize the actual average number of hits.

From this figure, it can be seen that the primitive roots selected with the metric 


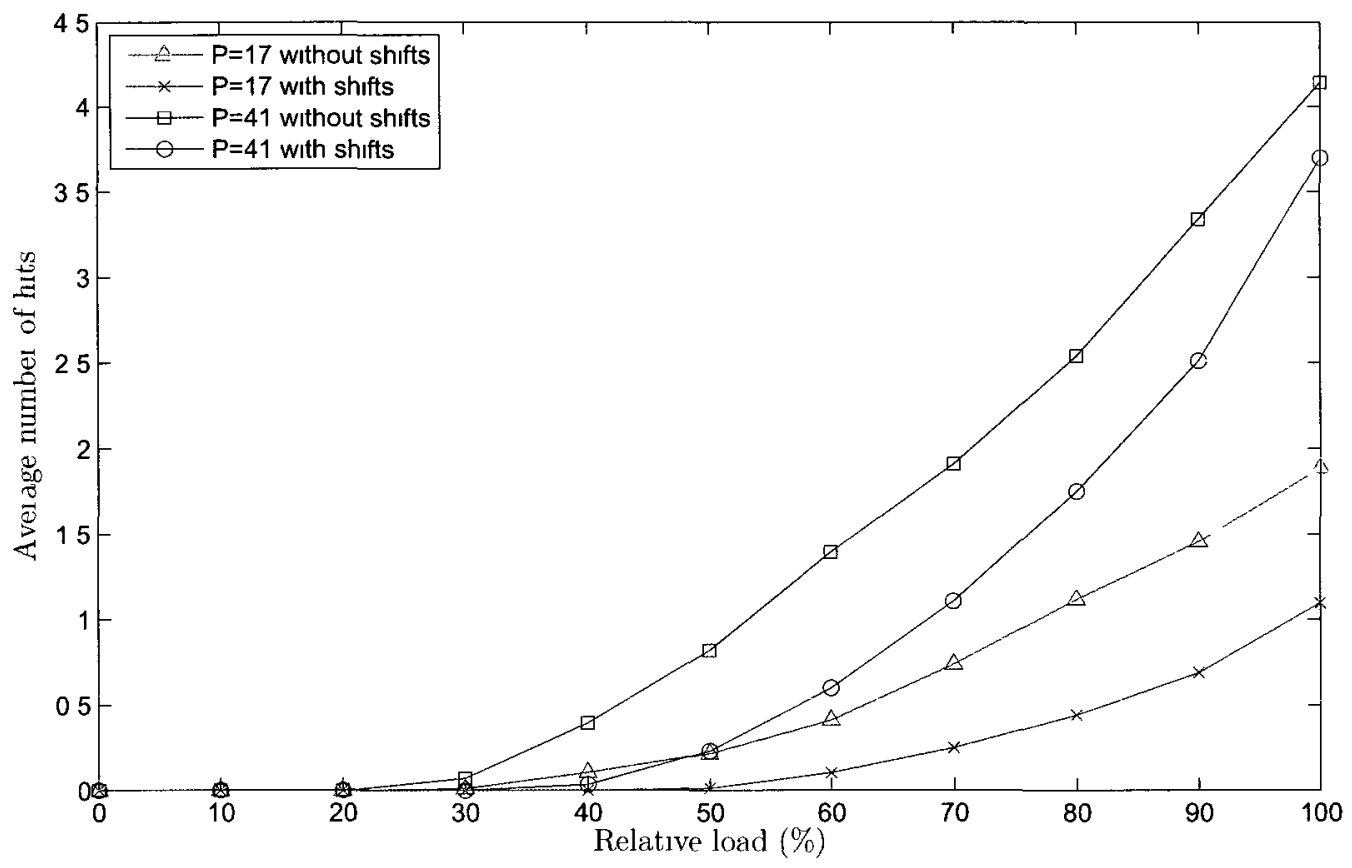

Figure 7.4: Proposed assignment scheme for $P=17$ and $P=41$ with and without cyclic shifts with immediate HIA.

yield close-to-optimal performance when $P$ is small. However, as $P$ increases, the performance of these roots exhibit a substantial deviation from that of the optimal ones.

Example 7. In this example, we show the performance gain resulting from incorporating cyclic shifts in the design of the assignment sequences. We consider the case in which $P=17$ and $P=41$ and the HIA technique is used assuming immediate hit source identification.

From Figure 7.4, it can be seen that, in comparison with the case in which the cyclic shifts are fixed, the joint optimization of shifts and primitive roots yields a significant reduction in the average number of hits. For instance, when $\frac{K}{P-1}=90 \%$ using cyclically shifted assignment sequences with HIA results in an average of 0.69 
and 2.5 hits for $P=17$ and $P=41$, respectively, whereas when no shifts are employed, the corresponding number of hits is 1.46 and 3.34, respectively. Hence, the additional degrees of freedom offered by incorporating cyclic shifts in the sequence design yield valuable performance benefits. However, to use the HIA technique in this case, each TR must know, not only the primitive roots of other TRs, but also their cyclic shift.

Example 8. In this example, we investigate the performance of the proposed RB assignment sequences for a system with three TRs and $N=16$ RBs. We compare the case in which the PRCS pairs are chosen to minimize $\frac{1}{N} \sum_{K=1}^{N} C(K)$ with the case in which the PRCS pairs are chosen to minimize $C(K)$ for each system load, $K$. In both cases, the HIA technique is employed assuming immediate hit source identification. From Figure 7.5, it can be seen that minimizing $C(K)$ at each $K$ yields marginal reduction in the average number of hits. For instance, for this example, the maximum reduction is less than 0.06 hits. Hence, it can be seen that minimizing $C(K)$ at each $K$ results in an average performance that is close to that obtained by minimizing $\frac{1}{N} \sum_{K=1}^{N} C(K)$, and does not warrant the additional complexity required for its implementation.

Example 9. In this example, we compare the average signal-to-noise-plus-interference ratio (SINR) achieved by the RB assignment sequences generated by (5.1), with the average SINR achieved by the random sequences proposed in [11] when the channel gains between the TRs and the WTs are assumed to be frequency-flat with complex Gaussian distribution of unit variance per real dimension. We consider a system with three TRs and $N=16 \mathrm{RBs}$. The loads of the first and second TRs are $k_{1}=2$ and $k_{2}=3$. The load of third TR $k_{3}$ is increased from 1 to 11 WTs. The transmission power of all TRs is set to be $10 \mathrm{~dB}$ and the noise variance is assumed to be unity. 


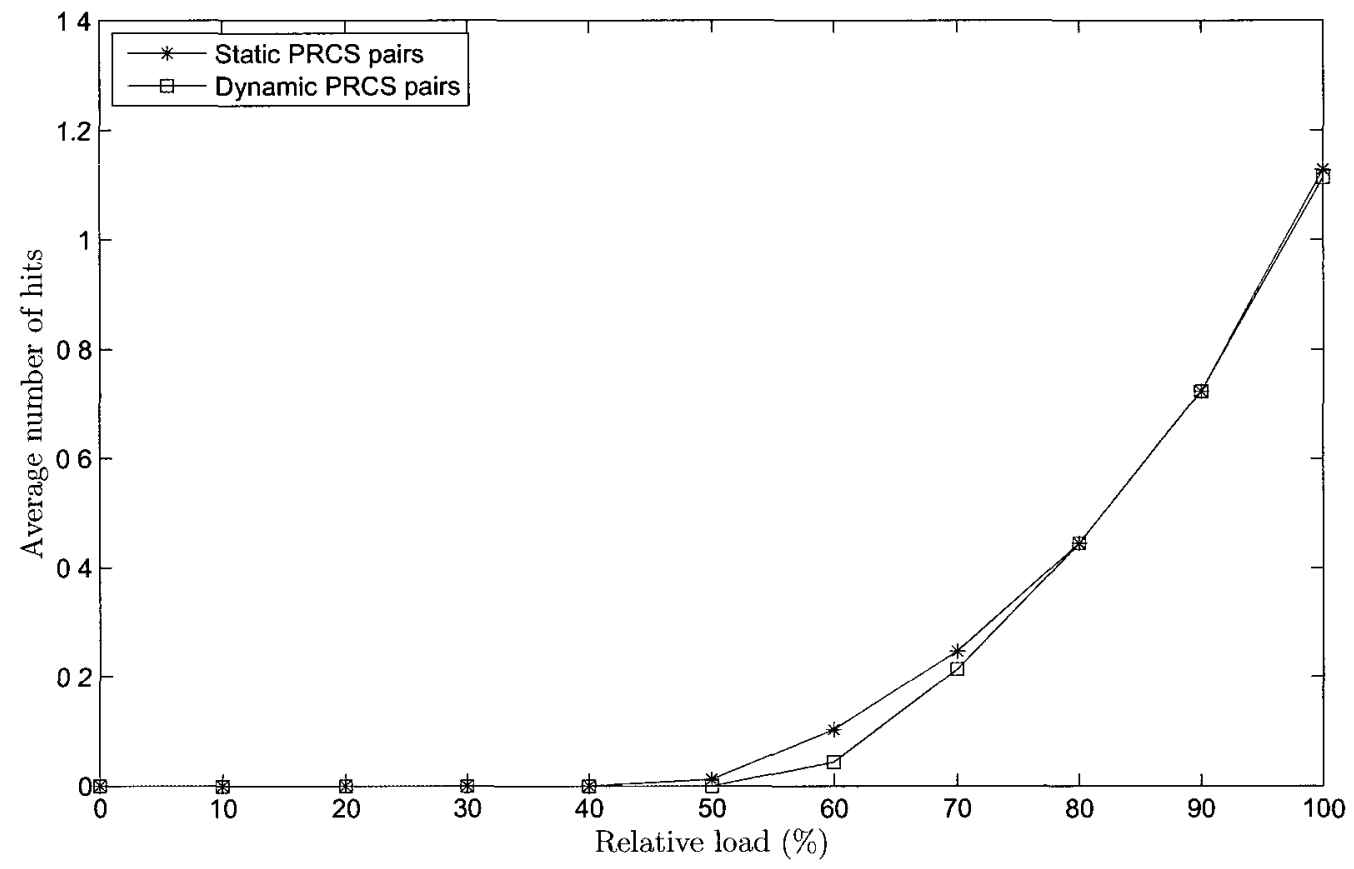

Figure 7.5: Comparison between the performance of the PRCS pairs that minimize $\frac{1}{N} \sum_{K=1}^{N} C(K)$ and that of the PRCS pairs that minimize $C(K)$ for each system load $K$. The number of RBs $N=16$. 


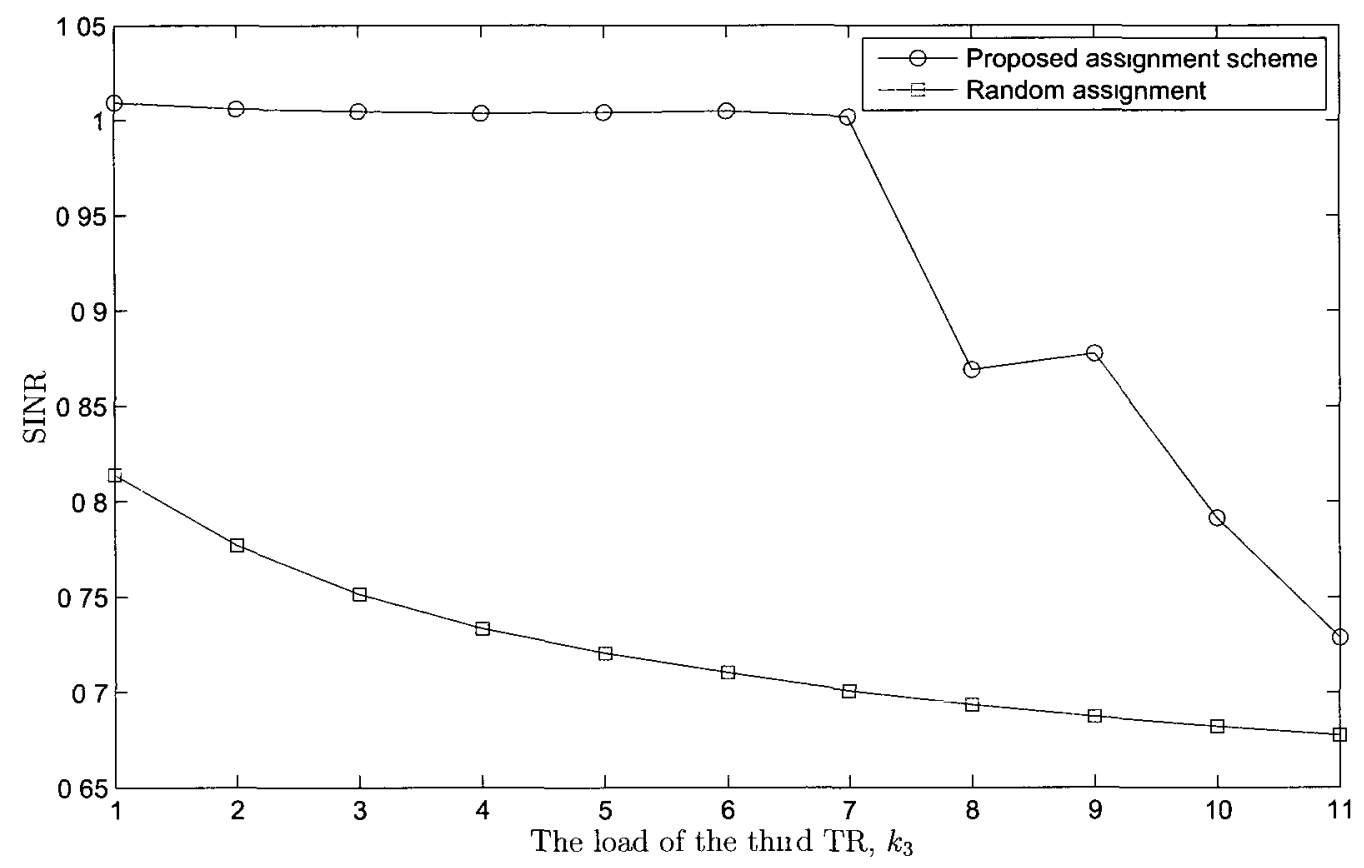

Figure 7.6: Comparison between the SINR achieved by the proposed RB assignment sequences and the random assignment sequences [11] for $N=16$ and $M=$ 3.

The SINR is averaged over 5000 channel realizations.

From Figure 7.6, it can be seen that the proposed RB assignment sequences can provide an average SINR performance improvement in excess of $30 \%$ over random assignments. While the average SINR of the random assignments decrease steadily with the load increase, three regions can be identified in the performance of the proposed sequences. In particular, for these sequences when $k_{3} \leq 7$, no hits occur, yielding a constant average SINR. For $k_{3}=8$, one hit occurred resulting in reducing the average SINR. However, when $k_{3}$ is increased to 9, no additional hits occur, which yields a slight increase in the average SINR. Further increase of $k_{3}$ results in hit occurrences, which reduce the average SINR.

Example 10. In this example, we compare the average achievable data rate by the 


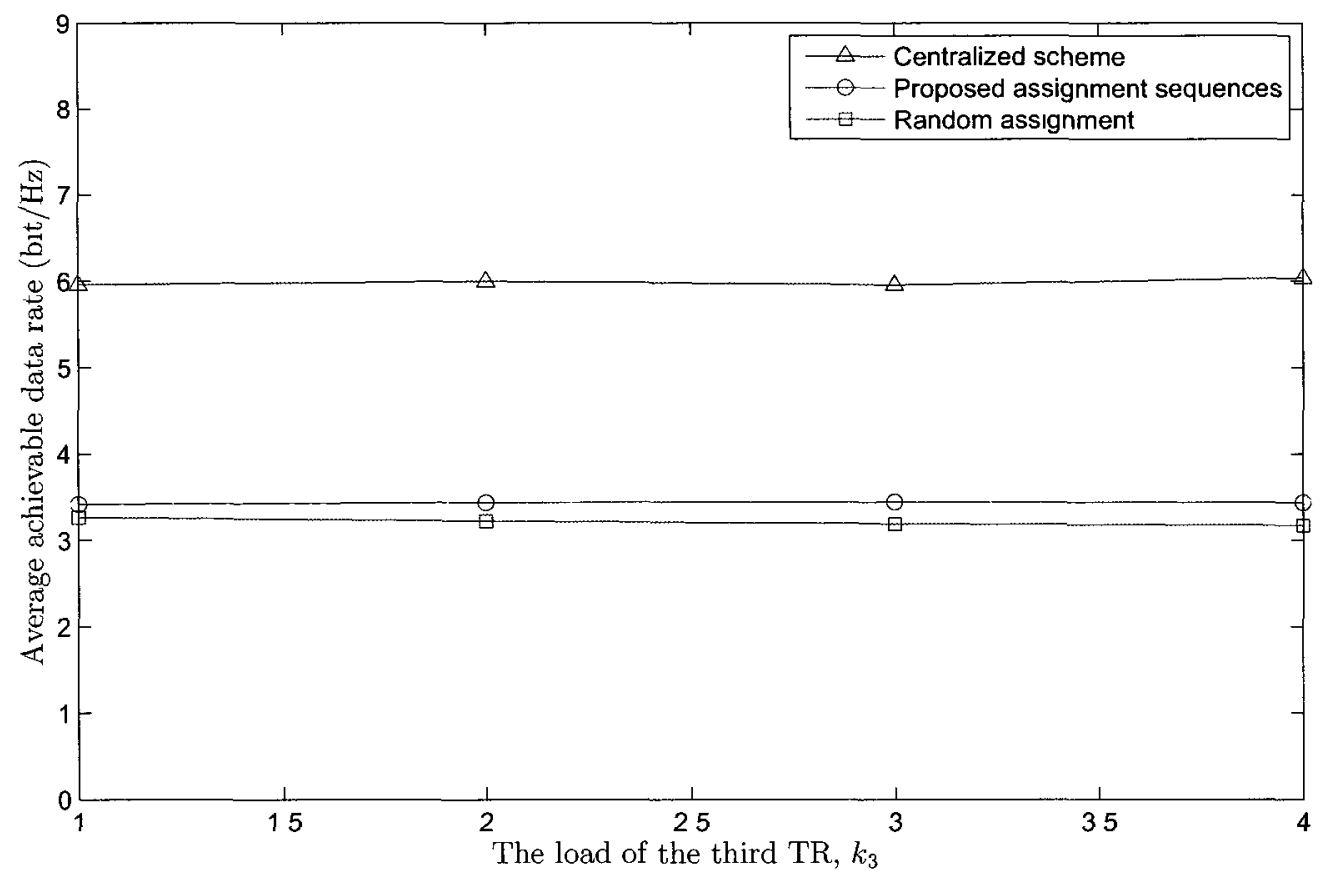

Figure 7.7: Comparison between the achievable data rate when the proposed RB assignment sequences, the random assignment sequences [11] and the optimal centralized assignment scheme with perfect CQI knowledge are employed for $N=16$ and $M=3$.

proposed sequences with an upper bound for a system similar to the one in the previous example. The transmission power of all TRs is set to be $10 \mathrm{~dB}$ and the noise variance is assumed to be unity. The upper bound is achieved by an optimal centralized scheme in which the BS has perfect CQI information and performs an exhaustive search over all possible RB assignments. The loads of the first and second TRs are $k_{1}=1$ and $k_{2}=2$, respectively. The load of the third TR, $k_{3}$, is increased from 1 to 4 WTs; assessing the performance of the optimal centralized scheme is computationally difficult for systems with more WTs. For comparison, we also show the average achievable data rate obtained by the random sequences proposed in [11].

As shown in Figure 7.7, the centralized scheme provides a performance superior 
to both the proposed scheme and the random one. However, the implementation of the centralized scheme requires the BS to acquire perfect CQI information about all TR-WT links and to perform a computationally prohibitive search for each channel realization.

The quality-of-service (QoS) that can be delivered by a system depends on the average SINR observed by the WTs. In particular, a minimum average SINR threshold must be exceeded to ensure the achievability of a target QoS. Since the average SINR tends to decrease when more WTs access the system, the average SINR threshold provides a trade-off between the QoS and the number of WTs that can be accommodated by the available RBs.

Example 11. In this example, the relative load, $K / N$, that can be accommodated in a three TR system for different average SINR thresholds is compared when the proposed and random assignment sequences are used. Similar to the previous example, the TR-WT channel gains are assumed to be frequency-flat with complex Gaussian distribution of unit variance per real dimension, and the number of RBs, $N=16$. The transmission power of all $\mathrm{TRs}$ is set to be $10 \mathrm{~dB}$ and the noise variance is set to be unity. The threshold SINR is averaged over 5000 channel realizations. To investigate the potential of $\mathrm{RB}$ reuse, in this example, $K / N$ is allowed to exceed $100 \%$. The loads of the first and the second TRs are $k_{1}=2$ and $k_{2}=3$, respectively, and the load of the third TR $k_{3}$ is increased from 1 to 16.

From Figure 7.8, it can be seen that, in comparison with random sequences, the proposed sequences result in a significant increase in the relative load that can be accommodated by the network, without compromising the QoS. For instance, at an average threshold SINR of 9.03, the system with random sequences accommodates 


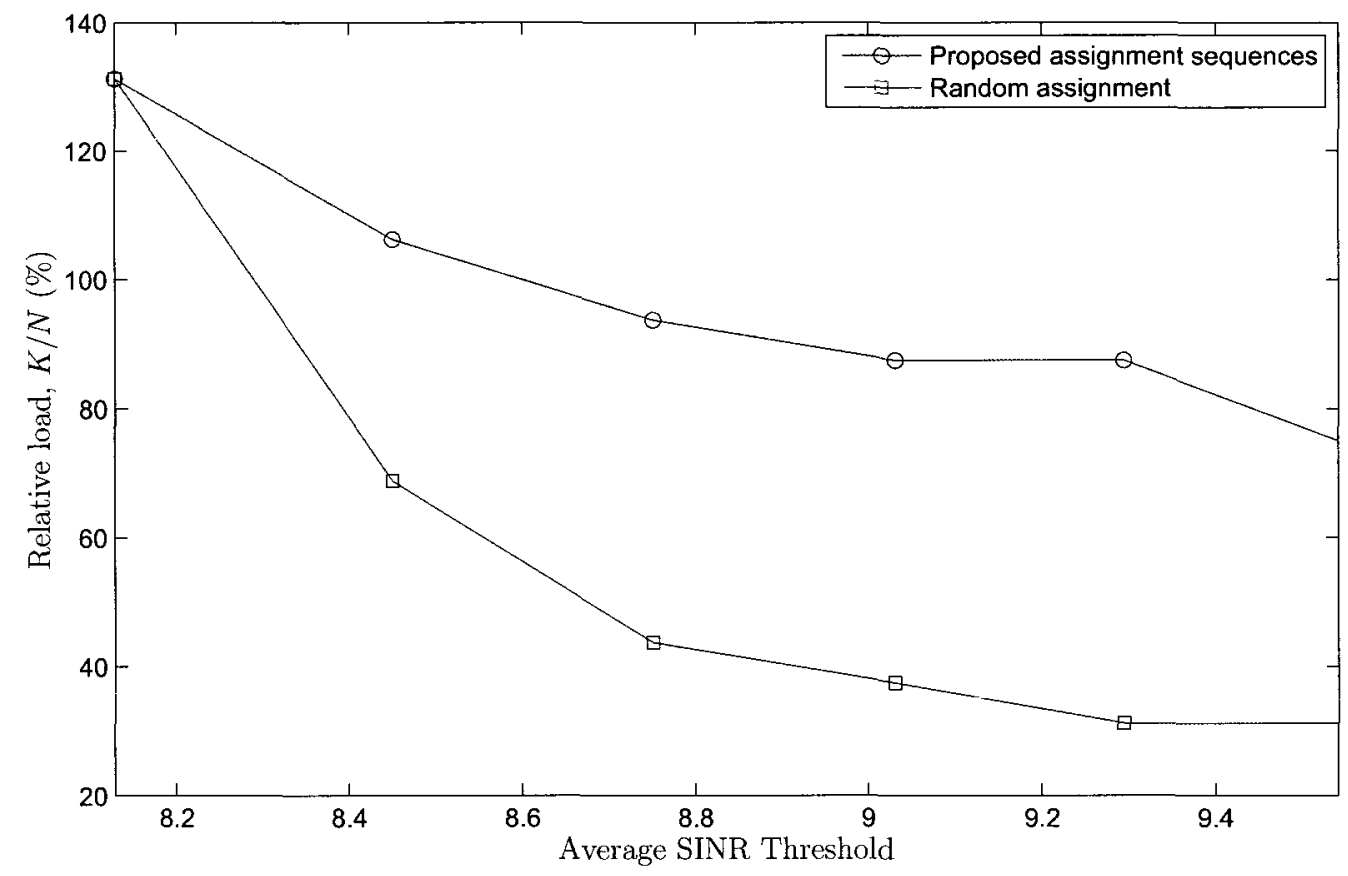

Figure 7.8: Comparison between the relative load of the random and proposed assignment sequences at different average threshold SINR levels for $N=16$ RBs.

$K / N=37.5 \%$, whereas that with the proposed sequences accommodates $K / N=$ $87.5 \%$; a gain of $233 \%$ in the system user capacity. This gain is attributed to the lower interference levels yielded by the proposed sequences.

PN sequences have been traditionally used for generating hopping patterns in $\mathrm{FH}$ systems. Similar to those systems, the decimal representation of the binary states of the PN sequences can be used to generate assignment sequences for the relayassisted OFDMA-based systems considered herein. The set of states generated by the PN sequences possess a cyclic group structure that facilitates their generation. In particular, for a given integer, $n>1$, there are $\frac{\phi\left(2^{n}-1\right)}{n}$ primitive polynomials that generate length- $\left(2^{n}-1\right)$ periodic PN sequences [26]. Notice that the length of the 


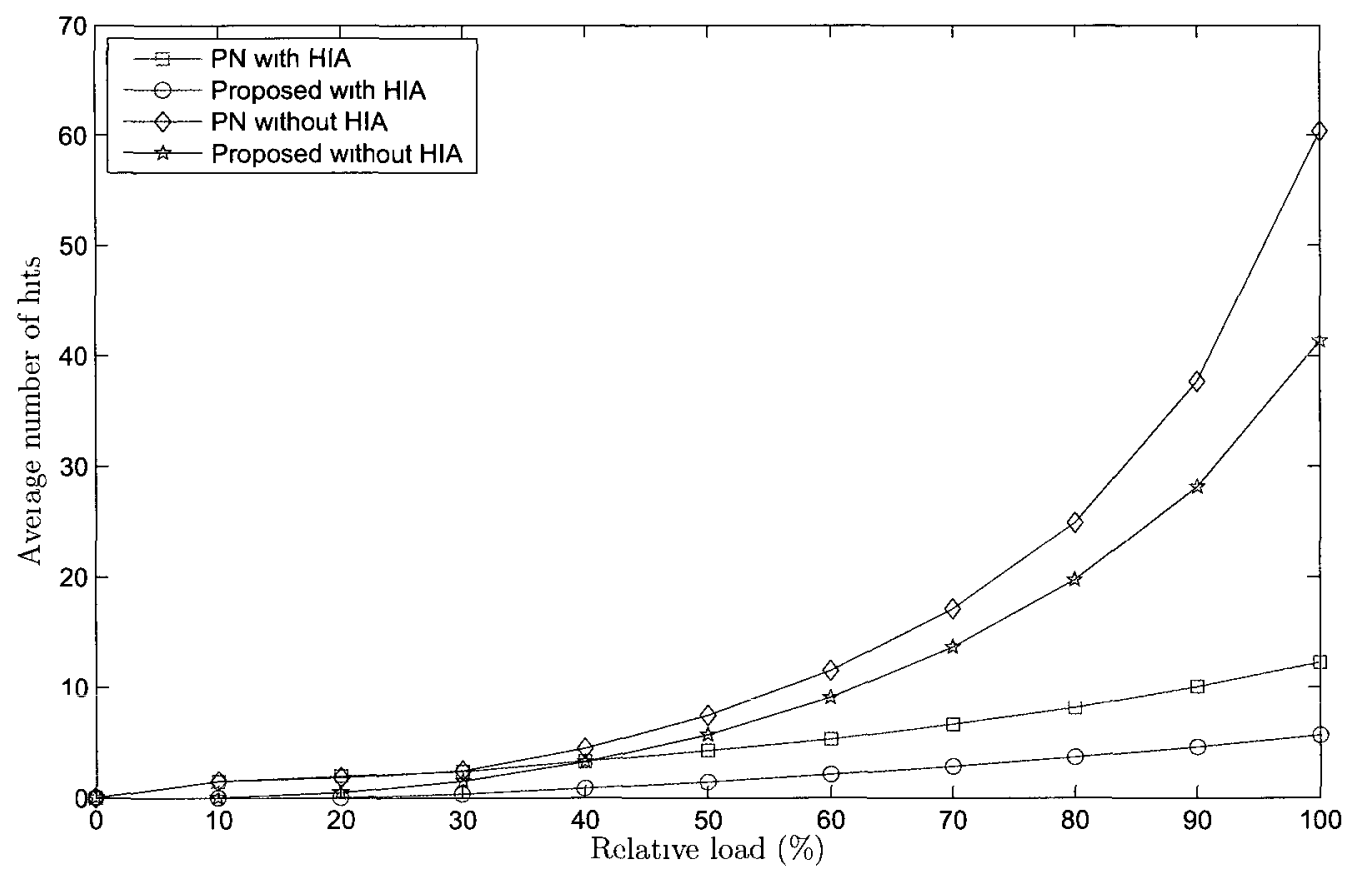

Figure 7.9: Comparison between the PN-based and the proposed assignment schemes for $P=127$ and $n=7$ when the cyclic shifts are set to zero with and without immediate HIA.

sequence generated by this technique is odd, whereas that generated by the proposed scheme is even. Hence, for comparison, we will consider prime integers of the form $P=2^{n}-1$, which yield sequence lengths that differ by one.

Example 12. In this example, we compare the performance of the $\mathrm{PN}$ sequences generated by the primitive polynomials that yielded the minimum number of hits with $n=7$ and the performance of the proposed assignment sequences with $P=127$. For simplicity, for the PN-based sequences, the initial state is chosen to be one, and for the proposed sequences the shifts are set to be zero. For both cases, we consider the performance of the assignment sequences when the HIA technique of Chapter 5 is employed. From Figure 7.9, it can be seen that the proposed assignment sequences yield a smaller average number of hits than their PN-based counterparts. 
For instance, when $\frac{K}{P-1}=90 \%$ the proposed scheme yields 4.6 hits where the PNbased assignment yields 10.1 hits. This performance improvement is attributed to the fact that the proposed scheme spans a set of sequences larger than that spanned by the PN generation technique.

Finally, it is to be noted that, because primes are denser than numbers of the form $2^{n}$ in the set of integers [23], the proposed scheme covers a selection of $N=|\mathcal{R}|$ larger than that covered by the PN-based assignment scheme; see Chapter 4 . 


\section{Chapter 8}

\section{Summary and Future Work}

In this thesis, we addressed the problem of RBs assignment for wireless networks that are assisted by terminal relays. In particular a novel technique for autonomous RBs assignment in relay-assisted OFDMA-based cellular networks is proposed. This technique eliminates the need for coordination between the TRs, and hence is particularly suited for emerging terminal relaying networks as it minimizes the coordination overhead. We conclude this thesis by highlighting the key points in our proposed work and introducing the open research directions for future work.

\subsection{Thesis Summary}

The key points in the proposed work hereinabove can be presented by the following items:

- In this thesis, we introduce an autonomous RB assignment scheme. In our scheme, the assignment sequences are endowed with a cyclic group structure. Hence, the sequences can be easily generated from a single PRCS pair. This results in a negligible coordination overhead in the case when the PRCS pairs have to be injected to the WTs by the BS. Moreover, the proposed RBs assignment scheme does not depend on the availability of the CQIs which may not be practical to acquire in a real time system. 
- In our proposed scheme, each relay independently assigns its resources based on its assigned or locally selected PRCS pair. However, due to the cyclic structure adopted by our scheme, each TR has access to all the available set of RBs. Hence our scheme is also suitable for the cases where we have non uniform user distributions.

- Given the cyclic structured assignment of our scheme, information regarding the load levels of neighboring relays can be extracted when a hit occurs. This allows the adjustments of the assignment sequences in order to improve the observed performance by reducing the number of hits. For this purpose, in Chapter 5, we propose a hit identification and avoidance algorithm that can perform this task on the fly due to its low computational complexity.

- Encouraged by the large number of the available combinations of PRCS pairs, we propose a metric for faster selection of the PRCS pairs in Chapter 5. This work was further extended by proposing a graphical PRCS pairs selection technique in Chapter 6.

- In Chapter 7 , we compared the performance of our proposed cyclically structured assignment sequences with the cases where uniformly distributed random assignment and PN based assignment sequences are employed. It was shown through simulations that our proposed scheme outperforms the random and PN based assignments with and without the employment of the HIA algorithm. 


\subsection{Proposed Future Work}

In this section we introduce our envisioned future work to further extend the proposed work hereinabove. The following bullets summarize the major directions and their expected performance outcome:

- In our proposed HIA algorithm, it was assumed that the TRs are able to identify the occurrence of a hit when an RB is assigned concurrently by two TRs. However, due to channel effects such as shadowing a TR might not be able to detect the occurrence of a hit. This will affect the logical procedure of the HIA algorithm and hence the HIA algorithm might fail in identifying the hit source. To mitigate this drawback, we propose an improvement of the HIA algorithm by exploiting the Trellis diagram concept. With this decoding technique employed, the TR will be able to identify the closest logical path and hence avoid misidentification of the hit source.

- In Chapter 6, we propose a graphical PRCS pairs selection technique to enable fast selection of the PRCS pairs. However we were only able to solve the problem of PRCS selection for up to a maximum of $M=4$ TRs. To extend this work, we propose further improvement of the proposed algorithm by employing a 3 dimensional graphical presentation of the PRCS pairs. In this case, we expect to have better insights on the performance of the PRCS pairs and hence can perform the graphical selection process for a larger number of TRs $M>4$.

- In this thesis, we assume that the TRs are not capable of sensing the spectrum before assigning the RBs to the WTs. While this assumption has its advantages in reducing the consumed power and eliminating the delay imposed by 
the sensing process, we would like to investigate the effect of employing occasional spectrum sensing on the performance of the proposed HIA algorithm. In particular, when the relays sense the spectrum they will be able to identify the RBs that are occupied by other TRs. Hence the TRs will be able to apply a modified version of the HIA algorithm to identify the load levels of neighboring relays and avoid future hits accordingly. 


\section{Appendix A}

\section{Proof of Lemma 1}

We will prove this lemma by contradiction.

Let $g_{1}$ and $g_{2}$ be two distinct generators of the group $G$. Suppose that the sequence generated by $g_{1}$ is a cyclically shifted version of the sequence generated by $g_{2}$. In this case we can write

$$
g_{1}^{k} \equiv g_{2}^{k+r} \quad(\bmod P)
$$

where $\equiv$ denotes congruence, $k \in\{1, \ldots, P-1\}$ and $r \in\{1, \ldots, P-2\}$ is the cyclic shift. By squaring both sides of (A.1) we have

$$
g_{1}^{2 k} \equiv g_{2}^{2 k+2 r} \quad(\bmod P)
$$

Consider the sequence generated by $g_{1}$. The entry of this sequence that lies after $k$

slots from $g_{1}^{k}(\bmod P)$ is $g_{1}^{2 k}(\bmod P)$. Using the assumption that this sequence is a cyclically shifted version of that generated by $g_{2}$, we have from (A.1) that

$$
g_{1}^{2 k} \equiv g_{2}^{2 k+r} \quad(\bmod P)
$$

Equating the right hand sides of (A.2) and (A.3) yields

$$
g_{2}^{2 k+2 r} \equiv g_{2}^{2 k+r} \quad(\bmod P)
$$


Multiplying both sides of (A.4) by $g_{2}^{p-1-2 k-2 r}$, it follows from Fermat's Little Theorem [23] that

$$
1 \equiv g_{2}^{p-1-r} \equiv g_{2}^{-r} \quad(\bmod P)
$$

The last equation can be satisfied if and only if $r=0(\bmod P-1)$. Using this in (A.1) and simplifying yields $g_{1}=g_{2}$, which contradicts the assumption that $g_{1}$ and $g_{2}$ are distinct, which completes the proof of the lemma. 


\section{Appendix B}

\section{Proof of Lemma 2}

As illustrated in Chapter 5, only the pair-wise hit matrix $H_{\imath \jmath}$ contribute to the number of hits observed between the assignment sequences of the two TRs, $i$ and $j$. The entries of this metric are given by the inner product of the load matrices $X_{\imath}$ and $X_{\jmath}$ which represent the sequences adopted by relays $i$ and $j$ respectively. However all cyclic groups of the same order are isomorphic regardless of the group operation where group isomorphism is a relabeling function as illustrated in Chapter 6 . This implies that the locations of the intersections between the sequences will be preserved. Hence, the observed number of hits will be the same. We will particularize this lemma by considering the case where we have additive and multiplicative cyclic groups. This particular case of the lemma can be proven as follows:

Let us consider the two groups $G_{1}$ and $G_{2}$ where $G_{1}$ is a multiplicative cyclic group and $G_{2}$ is an additive cyclic group. Both groups have the same order $P-1$. Let $g_{1}$ and $g_{2}$ be two distinct group generators of the multiplicative group $G_{1}$. We will denote the isomorphic mapping function from $G_{1}$ to $G_{2}$ by $\mathcal{F}$ where $\mathcal{F}\left(g_{1}^{k}\right)=k$ $(\bmod P-1)$. We note that the input of the mapping function $\mathcal{F}$ has to be in the form of powers of $g_{1}$. Similarly, let $h_{1}$ and $h_{2}$ be two group generators of the additive group $G_{2}$ where $h_{1}$ and $h_{2}$ are the corresponding elements from the mapping of $g_{1}$ and $g_{2}$ under the isomorphic mapping function $\mathcal{F}$. 
For the multiplicative group $G_{1}$, applying Equation 3.2 on the group generators $g_{1}$ and $g_{2}$ yields the sequences $s_{1}$ and $s_{2}$ as follows:

$$
\begin{aligned}
& s_{1}=\left\{g_{1}^{1}(\bmod P), g_{1}^{2}(\bmod P), \ldots, g_{1}^{P-1} \quad(\bmod P)\right\} . \\
& s_{2}=\left\{g_{2}^{1}(\bmod P), g_{2}^{2} \quad(\bmod P), \ldots, g_{2}^{P-1}(\bmod P)\right\} .
\end{aligned}
$$

Applying the mapping function $\mathcal{F}$ on the elements of B.1, yields

$$
s_{1}=\{1,2, \ldots, 0\}
$$

Given that the sequence generated by each group generator spans the complete sequence of integers from 1 to $P-1$, then there exists an integer $x$ such that $g_{2}=g_{1}^{x}$ $(\bmod P)$ where $x$ is an integer $0<x<P$. By substitution in B.2, the sequence $s_{2}$ then becomes:

$$
s_{2}=\left\{g_{1}^{x} \quad(\bmod P), g_{1}^{2 x} \quad(\bmod P), \ldots, g_{1}^{(P-1)(x)} \quad(\bmod P)\right\} .
$$

Similar to B.3, applying the mapping function $\mathcal{F}$ on B.4 yields

$$
s_{2}=\{x \quad(\bmod P-1), 2 x(\bmod P-1), \ldots,(P-1)(x) \quad(\bmod P-1)\} .
$$

Applying $\mathcal{F}$ on $g_{1}$ and $g_{2}$ yields the following values for $h_{1}$ and $h_{2}$ :

$$
h_{1}=\mathcal{F}\left(g_{1}\right)=1, \text { and } h_{2}=\mathcal{F}\left(g_{2}\right)=\mathcal{F}\left(g_{1}^{x} \quad(\bmod P)\right)=x .
$$

For the additive group $G_{2}$, applying Equation 3.1 on the group generators $h_{1}$ and $h_{2}$ yields the sequences $s_{3}$ and $s_{4}$ as follows:

$$
\begin{aligned}
& s_{3}=\left\{(1)\left(h_{1}\right) \quad(\bmod P-1), 2 h_{1}(\bmod P-1), \ldots,(P-1)\left(h_{1}\right) \quad(\bmod P-1)\right\} . \\
& s_{4}=\left\{(1)\left(h_{2}\right) \quad(\bmod P-1), 2 h_{2} \quad(\bmod P-1), \ldots,(P-1)\left(h_{2}\right) \quad(\bmod P-1)\right\} .
\end{aligned}
$$


Substituting from B.6 in B.7 and B.8 yields:

$$
\begin{gathered}
s_{3}=\{1,2, \ldots, 0\} \\
s_{4}=\{x \quad(\bmod P-1), 2 x(\bmod P-1), \ldots,(P-1)(x) \quad(\bmod P-1)\} .
\end{gathered}
$$

From Equations B.1, B.2, B.9, and B.10, it can be seen that both groups will generate the same sequences regardless of their group operation. 


\section{References}

[1] "Mobile broadband the benefits of additional spectrum," Tech. Rep. 6, Federal Communications Commission, Oct 2010.

[2] T. S. Rappaport, Wireless Communıcatıons, Prıncıples and Practıce. New Jersey: Prentice Hall, 2002.

[3] L. Le and E. Hossain, "Multihop cellular networks: Potential gains, research challenges, and a resource allocation framework." IEEE Commun. Mag., vol. 45, pp. $66-73$, Sept. 2007.

[4] V. Sreng, H. Yanikomeroglu, and D. Falconer, "Coverage enhancement through two-hop relaying in cellular radio systems," in IEEE Wireless Commun. and Networking Conf. (WCNC), Orlando, Mar. 2002.

[5] R. Pabst, B. H. Walke, D. C. Schultz, P. Herhold, H. Yanikomeroglu, S. Mukherjee, H. Viswanathan, M. Lott, W. Zirwas, M. Dohler, H. Aghvami, D. D. Falconer, and G. P. Fettweis, "Relay-based deployment concepts for wireless and mobile broadband radio," IEEE Commun. Mag., vol. 42, pp. 80-89, Sept. 2004.

[6] A. R. Mishra, Fundamentals of Cellular Network Plannıng and Optımısatıon: 2G 2.5G 3G... Evolution to 4G. San Francisco: Wiley-Interscience, 2004.

[7] A. L. Stolyar and H. Viswanathan, "Self-organizing dynamic fractional frequency reuse in OFDMA systems," in Proc. IEEE Int. Conf. Comp. Commun. (INFOCOM), Phoenix, 2008.

[8] A. Nosratinia and T. E. Hunter, "Grouping and partner selection in cooperative wireless networks," IEEE J. Select. Areas Commun., vol. 25, pp. 369-378, Feb. 2007.

[9] T. C.-Y. Ng and W. Yu, "Joint optimization of relay strategies and resource allocations in cooperative cellular networks," IEEE J. Select. Areas Commun., vol. 25, pp. 328-339, Feb. 2007.

[10] K. Jitvanichphaibool, R. Zhang, and Y.-C. Liang, "Optimal resource allocation for two-way relay-assisted OFDMA," in Proc. IEEE Glob. Commun. Conf. (GLOBECOM), New Orleans, Dec. 2008. 
[11] C. Koutsimanis and G. Fodor, "A dynamic resource allocation scheme for guaranteed bit rate services in OFDMA networks," in Proc. IEEE Int. Conf. Commun. (ICC), Beijing, 2008.

[12] Z. Kostic and N. Sollenberger, "Performance and implementation of dynamic frequency hopping in limited-bandwidth cellular systems," IEEE Trans. Wireless Commun., vol. 1, pp. 28-36, Jan. 2002.

[13] P. Popovski, H. Yomo, S. Aprili, and R. Prasad, "Frequency rolling: A cooperative frequency hopping for mutually interfering WPANS," in Proc. ACM Int. Symp. Mobıle Ad Hoc Ntwk. Computing (MOBIHOC), Tokyo, May 2004.

[14] Z. Kostić, I. Marić, and X. Wang, "Fundamentals of dynamic frequency hopping in cellular systems," IEEE J. Select. Areas Commun., vol. 19, pp. 2254-2266, Nov. 2001.

[15] O. Mubarek, H. Yanikomeroglu, and S. Periyalwar, "Dynamic frequency hopping in cellular fixed relay networks," in Proc. IEEE Vehıc. Tech. Conf. (VTC), vol. 5, Stockholm, May 2005.

[16] J. Huang, R. A. Berry, and M. L. Honig, "Distributed interference compensation for wireless networks," IEEE J. Select. Areas Commun., vol. 24, pp. 1074-1084, May 2006.

[17] R. Etkin, A. Parekh, and D. Tse, "Spectrum sharing for unlicensed bands," IEEE J. Select. Areas Commun., vol. 25, pp. 517-528, Apr. 2007.

[18] B. Babadi and V. Tarokh, "GADIA: A greedy asynchronous distributed interference avoidance algorithm," IEEE Trans. Inf. Theory, vol. 56, pp. 6228 6252, Dec. 2010.

[19] J. Huang, Z. Han, M. Chiang, and H. V. Poor, "Auction-based resource allocation for cooperative communications," IEEE J. Select. Areas Commun., vol. 26, pp. 1226-1237, Sept. 2008.

[20] J. Sangiamwong, T. Asai, J. Hagiwara, and T. Ohya, "Cooperative diversity aware priority-based resource allocation for relay-enhanced cellular systems," in Proc. IEICE Asıa-Pacıfic Conf. on Commun. (APCC), Tokyo, 2008.

[21] J. A. Gallian, Contemporary Abstract Algebra. Boston: Houghton Mifflin, 1998.

[22] T. W. Hungerford, Abstract Algebra: An Introduction. Philadelphia: Saunders College Publishing, 1997. 
[23] W. W. Adams and L. J. Goldstein, Introduction to Number Theory. New Jersey: Prentice-Hall, Inc., 1976.

[24] S. M. Ross, Stochastıc Processes. New York: John Wiley, 1996.

[25] G. E. Andrews, The Theory of Partıtıons. Cambridge: Cambridge University Press, 1976.

[26] R. Lidl and H. Niederreiter, Introduction to Finıte Fields and Their Applicatıons. Cambridge: Cambridge University Press, 1994. 